\title{
Cdk5-Mediated Phosphorylation of Sirt1 Contributes to Podocyte Mitochondrial Dysfunction in Diabetic Nephropathy
}

\author{
Shuo Wang, ${ }^{1,2, *}$ Yakun Yang, ${ }^{1,2, *}$ Xingyu He, ${ }^{1,2}$ Lin Yang, ${ }^{3}$ Jianrong Wang, ${ }^{3}$ Shunjie Xia,,2 \\ Dan Liu, ${ }^{1,2}$ Shuxia Liu, ${ }^{1,2}$ Li Yang, ${ }^{4}$ Wei Liu, ${ }^{1,2}$ and Huijun Duan ${ }^{1,2}$
}

\begin{abstract}
Aims: Mitochondrial dysfunction contributes to podocyte injury, which is the leading cause of proteinuria in diabetic nephropathy (DN). In this study, we explored the role of cyclin-dependent kinase 5 (Cdk5) in mitochondrial dysfunction of podocytes under diabetic conditions.

Results: Our results showed that the expression and activity of Cdk5 were significantly upregulated in vivo and in vitro under diabetic conditions, accompanied by the downregulation of synaptopodin and nephrin, as well as structural and functional mitochondrial dysfunction. Inhibition of Cdk5 with roscovitine or dominant-negative $\mathrm{Cdk} 5$ led to the attenuation of podocyte injury by upregulating synaptopodin and nephrin. The inhibition of Cdk5 also ameliorated mitochondrial dysfunction by decreasing reactive oxygen species levels and cytochrome c release, while increasing adenosine triphosphate production. Sirt1, an NAD ${ }^{+}$-dependent deacetylase, was decreased in podocytes with high glucose (HG) treatment; however, its phosphorylation level at S47 was significantly upregulated. We demonstrated that HG levels cause overactive Cdk5 to phosphorylate Sirt 1 at S47. Suppression of Cdk5 reduced Sirt1 phosphorylation levels and mutation of S47 to nonphosphorable alanine (S47A), significantly attenuated podocyte injury and mitochondrial dysfunction in diabetic condition in vivo and in vitro.

Innovation and Conclusion: Our study has demonstrated the role of Cdk5 in regulating mitochondrial function through Sirt1 phosphorylation and thus can potentially be a new therapeutic target for DN treatment. IRB number: 20190040. Antioxid. Redox Signal. 34, 171-190.
\end{abstract}

Keywords: diabetic nephropathy, mitochondrial dysfunction, podocyte, Cdk5, Sirt1

\section{Introduction}

A S ONE OF THE MOST COMMON and serious microvascular complications of diabetes, diabetic nephropathy (DN) is one of the primary causes of end-stage renal disease worldwide $(28,38)$. Podocytes are terminally differentiated epithelial cells of the kidney, and their body extends interdigitated foot processes (FPs) forming the crucial component of the glomerular filtration barrier that prevents protein leakage (39). Injury, dedifferentiation, detachment, and apoptosis of podocytes are pathological hallmarks of DN, which promotes proteinuria and eventually lead to glomerulosclerosis $(37,45)$.
Mitochondria are key organelles of cellular bioenergetics and constantly undergo dynamic remodeling processes, including fusion and fission to adjust to cellular demand for energy (6). Podocytes cannot regenerate after differentiation and maturation, and their proliferative and regenerative abilities are extremely limited. Therefore, many mitochondria are needed in their cytoplasm to provide energy for maintaining cell structure and function. Emerging evidence suggests that mitochondrial dysfunction influences the development and progression of DN, especially during podocyte injury $(41,49)$. Mitochondrial dysfunction induced by high glucose (HG) level, with excess reactive oxygen species (ROS) production, is a major reason

\footnotetext{
${ }^{1}$ Key Laboratory of Kidney Diseases of Hebei Province, Department of Pathology, Hebei Medical University, Shijiazhuang, China. China.

Departments of ${ }^{3}$ Nephrology and ${ }^{4}$ Cardiac Ultrasound, Second Hospital of Hebei Medical University, Shijiazhuang, China.

*These authors contributed equally to this work.
} 


\section{Innovation}

Mitochondrial dysfunction contributes to podocyte injury, which is the leading cause of proteinuria in diabetic nephropathy (DN). The mechanism of mitochondrial dysfunction in podocytes in diabetes remains to be fully elucidated. Our investigation reveals a novel role for cyclin-dependent kinase $5(\mathrm{Cdk} 5)$ in regulating mitochondrial function through Sirt1 phosphorylation. We found that $\mathrm{Cdk} 5$ contributions to podocyte mitochondria dysfunction are significantly dependent on its close interactions with Sirt1, thus phosphorylating Sirt1 in vitro and in vivo. Our findings unraveled prospects for the potential targeting of mitochondrial functions by modulating Cdk5 activities as a treatment strategy against DN.

for podocyte injury in diabetes $(42,58)$. The contribution and mechanism of mitochondrial dysfunction to DN onset or progression remain unclear, especially in podocyte injury. Studies have shown that ROS generation and oxidative stress caused by HG promote podocyte apoptosis $(15,42)$.

Cyclin-dependent kinase $5(\mathrm{Cdk} 5)$ is a proline-directed serine/threonine kinase that belongs to the family of cyclindependent kinases (Cdks) (8). Unlike other Cdk members, Cdk5 activity is primarily controlled by specific co-activators, p35 and p39 (24). The predominance of both Cdk5 activators and Cdk5 activity was initially discovered in neuronal cells and tissues (52). Cdk5 plays an important role in regulating central nervous system development and synaptic plasticity (10). However, deregulation of the Cdk5 activity has cytotoxic consequences in cells and is associated with mitochondrial dysfunction in neurons, a leading cause of the development of neurodegenerative diseases, such as Alzheimer's disease (AD), Parkinson's disease (PD), and Huntington's disease (36).

Recently, studies have found that Cdk5 is also present in multiple nonneuronal cells and tissues (33). It was predominantly detected in podocytes from the capillary loop stage up to maturation and persisted in the podocytes of adult glomeruli; it plays important roles in podocyte differentiation, proliferation, and morphology $(7,57)$. It has been demonstrated that HG and transforming growth factor- $\beta 1$ (TGF- $\beta 1$ ) induced podocyte apoptosis by increasing the expression of Cdk5 (55). Whether Cdk5 participates in podocyte injury by inducing mitochondrial dysfunction in DN has not been clarified.

Sirt1, a silent information regulator, is a member of the deacetylase family and is involved in physiological and pathological processes such as cell senescence, metabolism, and apoptosis (2). In the past decade, Sirt1 has been found to promote the steady-state energy remodeling of kidney cells in high glucose environments, regulate oxidative stress, resist renal cell apoptosis, inhibit kidney inflammation, and improve renal fibrosis during the onset of DN $(1,5,23,53)$. Thus, Cdk5 seems to be of special interest in the context of Sirt1 regulation. Studies have shown that Cdk5-mediated hyperphosphorylation of Sirt1 contributes to the development of endothelial senescence and atherosclerosis (4).

In this study, we investigated the direct interplay between Cdk5 and Sirt1 in podocyte injury under diabetic conditions. Importantly, we explored the novel role of $\mathrm{Cdk} 5$ in regulating mitochondrial function through phosphorylation of Sirt1, which could lead to immense opportunities to target mitochondrial function and Cdk5 in the treatment of DN.

\section{Results}

\section{Cdk5 is increased in the glomeruli of patients with $D N$ and diabetic mice}

To validate the expression level of $\mathrm{Cdk} 5$ in the glomerulus, the renal biopsies from patients pathologically diagnosed with DN were analyzed by immunohistochemistry. In control tissues, low level of Cdk5 was seen in the glomeruli, whereas significantly stronger expression of Cdk5 was detected in the glomeruli of patients with DN (Fig. 1A, B). In contrast to $\mathrm{Cdk} 5$, nephrin and synaptopodin were markedly downregulated in DN (Fig. 1C-F). Furthermore, statistical analysis demonstrated that the Cdk5 staining intensity negatively correlated with the intensity of nephrin and synaptopodin (Fig. 1G, H). Immunofluorescence double-labeling experiments indicated that $\mathrm{Cdk} 5$ colocalized with nestin, a marker of podocyte, in the glomerulus (Fig. 1I, J).

We next detected the expression of $\mathrm{Cdk} 5$ in diabetic mice. Compared with the control group, the immunohistochemical analysis showed that the expression levels of Cdk5 were significantly increased in streptozotocin (STZ)-induced diabetic mice and $\mathrm{db} / \mathrm{db}$ mice, along with the decreased expressions of nephrin and synaptopodin (Fig. 1K-R).

\section{HG upregulates the expression and activation of Cdk5 in human podocytes}

To explore the effects of hyperglycemia on Cdk5 expression, human podocytes were treated with $30 \mathrm{~m} M$ glucose for 0,12 , 24,48 , and $72 \mathrm{~h}$. As the markers of podocyte injury, the expression levels of nephrin and synaptopodin were significantly decreased by $\mathrm{HG}$ treatment (Fig. 2A-C). Upregulation of Cdk5 and TGF- $\beta 1$ was observed in podocytes after $12 \mathrm{~h}$ HG treatment, and the expression reached its highest level at 24 and $48 \mathrm{~h}$ (Fig. 2A, D, E). p25, cleaved from the Cdk5 activator p35, was also significantly upregulated after 12 and $24 \mathrm{~h}$ (Fig. 2A, F, G). Kinase activity analysis showed that the level of p-Histone $\mathrm{H} 1$ was significantly increased by $\mathrm{HG}$ treatment at $24 \mathrm{~h}$, which demonstrated that $\mathrm{HG}$ conditions may lead to overactive Cdk5

FIG. 1. Cdk5 increased in the glomeruli of patients with DN and diabetic mice. (A, C, E) Representative immunohistochemical staining for Cdk5, synaptopodin, and nephrin in the glomerulus. (B, D, F) Quantification of Cdk5-, synaptopodin-, and nephrin-positive area in the glomerulus. $(p<0.05, n=19)(\mathbf{G}, \mathbf{H})$ The correlation between Cdk5 with synaptopodin and nephrin levels in DN patients was evaluated by the Spearman correlation analysis. (I, J) Colocalization of Cdk5 and nestin in the glomerulus, DAPI is a nucleus dye. $(\mathbf{K}, \mathbf{L})$ Representative immunohistochemical staining for Cdk5, synaptopodin, and nephrin in the glomerulus from diabetic mice. (M-R) Quantification of Cdk5-, synaptopodin-, and nephrin-positive area in the glomerulus. $(p<0.05)$. Scale bars: $50 \mu \mathrm{m}$. Magnification: $400 \times$. Cdk5, cyclin-dependent kinase 5 ; DN, diabetic nephropathy. Color images are available online. 

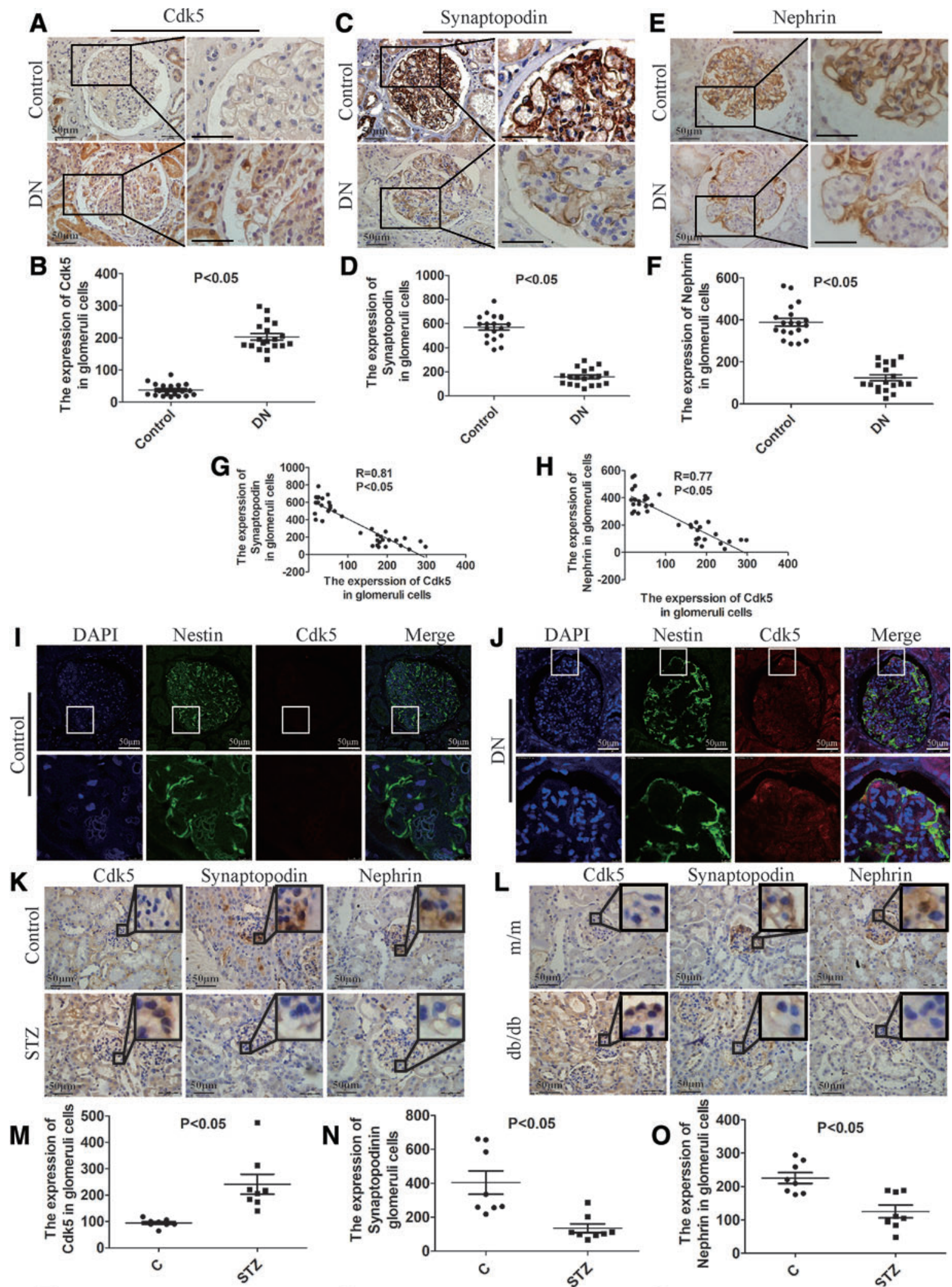

Nephrin
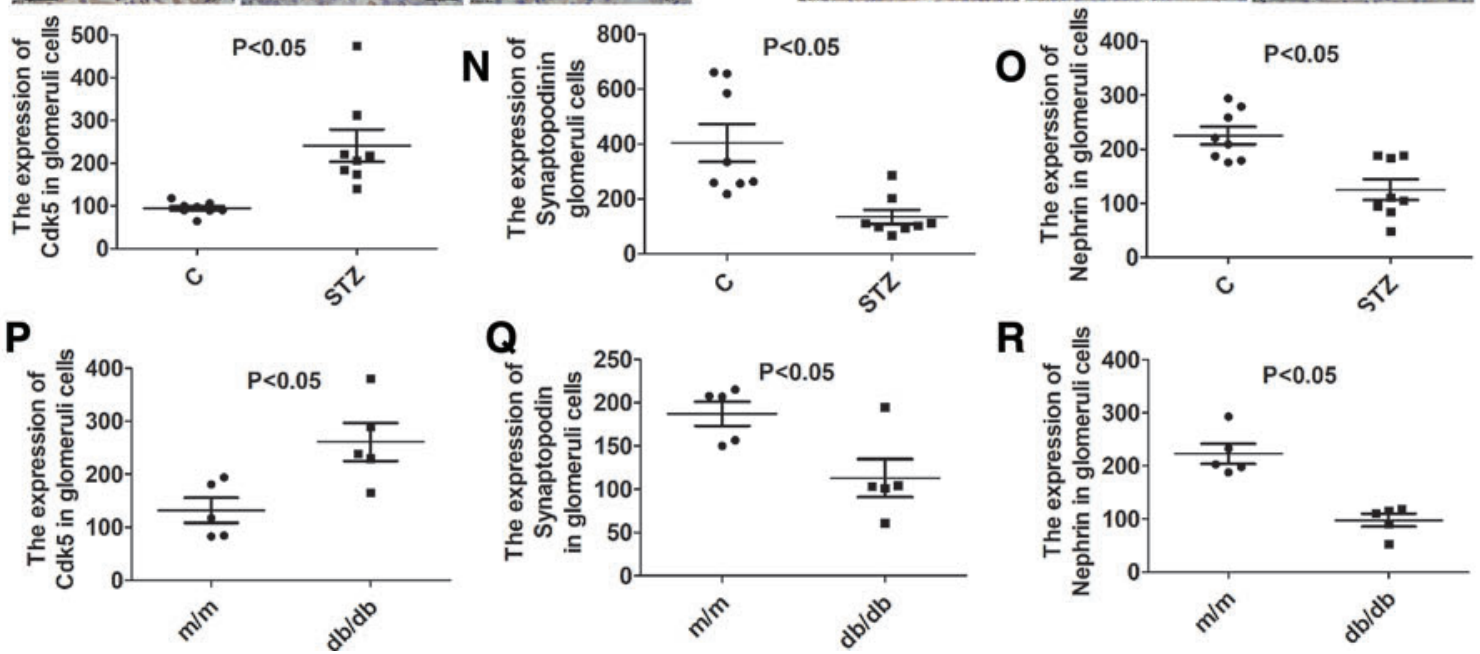

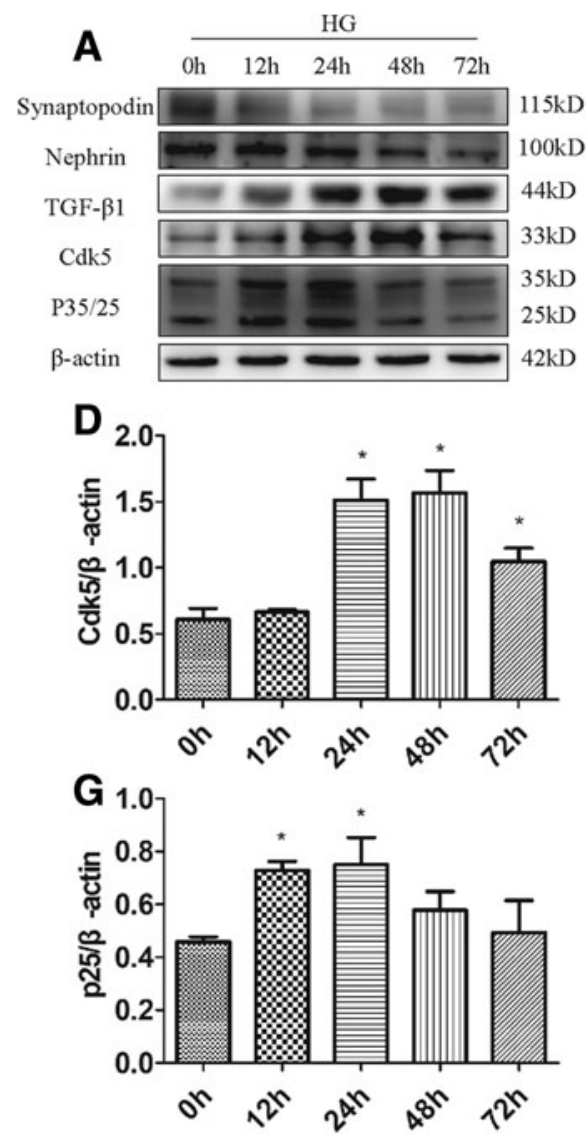

H
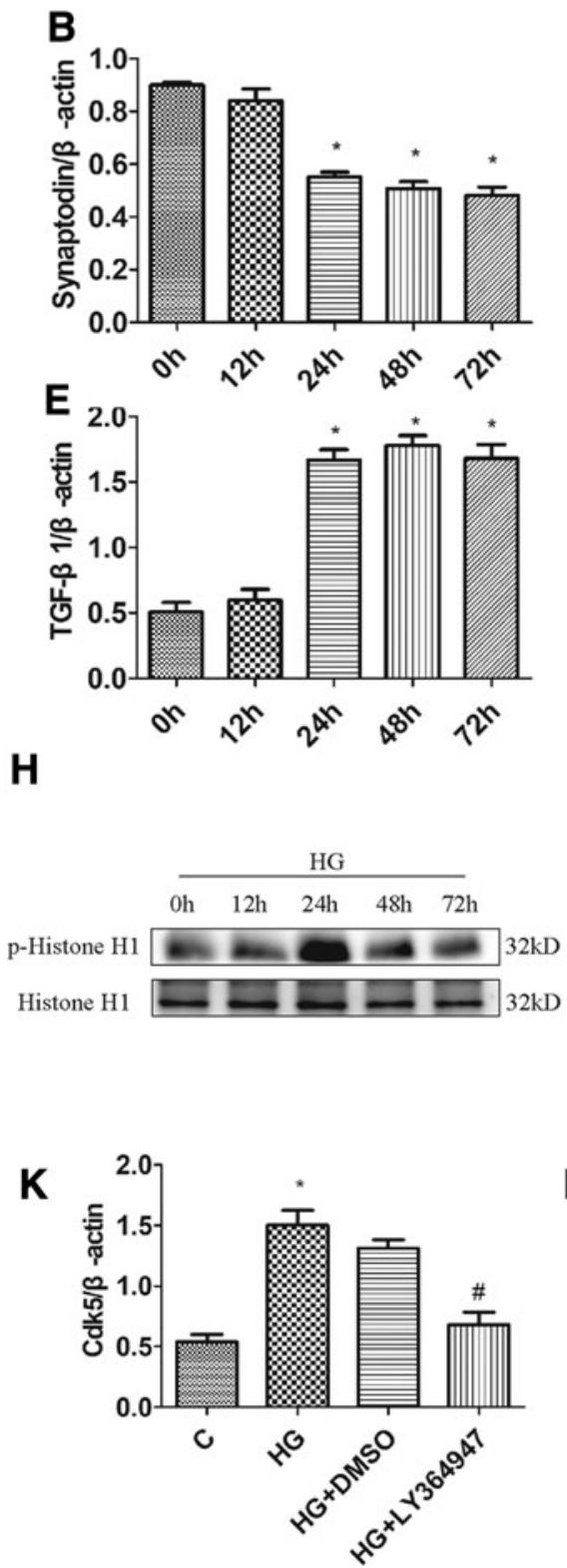

C
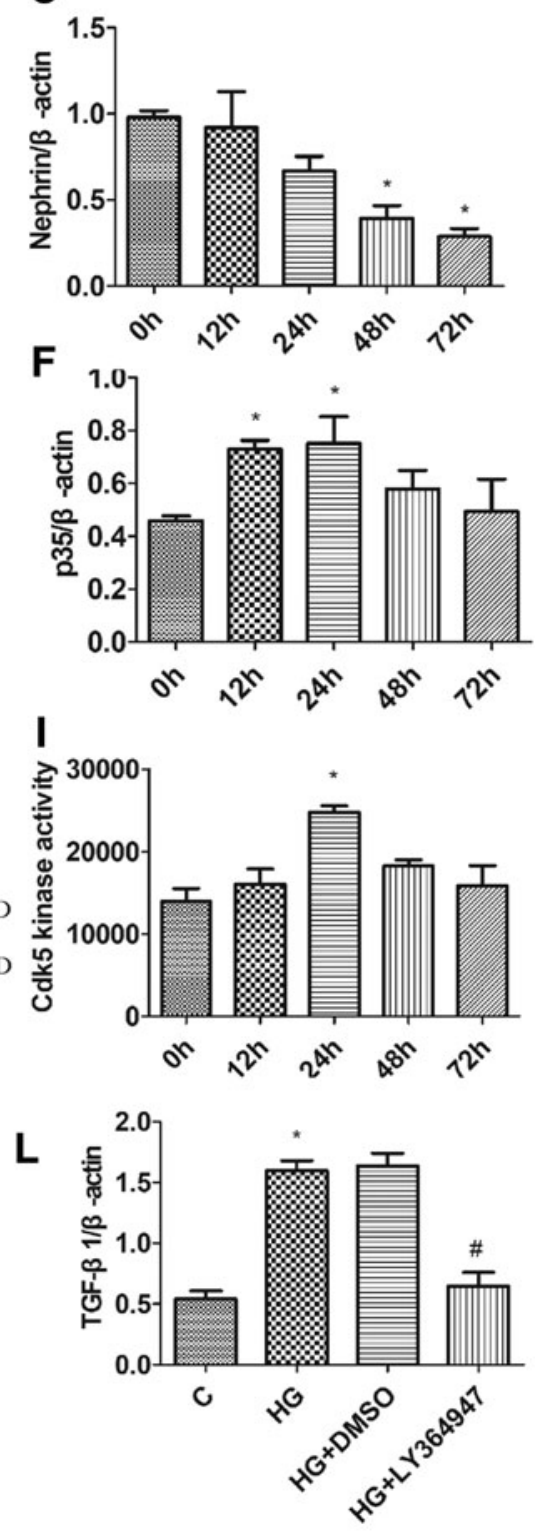

FIG. 2. HG upregulated the expression and activation of Cdk5 in human podocytes. (A) Protein representative Cdk5, P35/25, TGF- $\beta 1$, synaptopodin, and nephrin in HPCs induced by $30 \mathrm{~m} M$ glucose for different time points. (B-G) Protein levels of Cdk5, P35/ 25 , TGF- $\beta 1$, synaptopodin, and nephrin. (H) Protein representative photographs of Histone H1 and phospho-Histone H1 in HPCs induced by HG. (I) Protein levels of phospho-Histone H1. (J) Protein representative photographs of TGF- $\beta 1$ and Cdk5 in HPCs treated by LY364947 in $24 \mathrm{~h} \mathrm{HG}$ conditions. (K, L) Protein levels of TGF- $\beta 1$ and Cdk5.The data represent the mean $\pm \operatorname{SD}(n=3)$. p-, phospho-. ${ }^{*} p<0.05$ compared with the control group, ${ }^{*} p<0.05$ compared with the HG+DMSO group. Please refer to Supplementary Figure S1 for raw Western blot records shown in the figure. HG, high glucose; HPC, human renal podocyte.

(Fig. 2H, I). Human renal podocytes (HPCs) were treated with LY364947, an inhibitor of TGF- $\beta 1$, in $24 \mathrm{~h} \mathrm{HG}$ conditions, which caused a decrease in Cdk5 expression (Fig. 2J-L). These results verified that hyperglycemia, possibly via TGF- $\beta 1$, upregulates and activates Cdk5 (Supplementary Fig. S1).

\section{Cdk5 suppression attenuates HG-induced podocyte injury by alleviating mitochondrial dysfunction}

To confirm whether Cdk5 is directly associated with podocyte injury, roscovitine, a Cdk5 inhibitor, was used to suppress the Cdk5 kinase activity and expression. Roscovitine cytotoxicity was detected by CCK8. The results showed that the viability of cells was $<40 \%$ after treatment with $20 \mu M$ roscovitine (Fig. 3A). Thus, for subsequent experiments, we selected $2.5,5$, and $10 \mu M$ roscovitine to treat podocytes. As shown in Figure 3, treatment with roscovitine significantly recovered the expression of nephrin and synaptopodin, which decreased significantly after HG stimulation, as detected by Western blotting, quantitative real-time polymerase chain reaction (RT-qPCR), and immunofluorescence. These results demonstrated that the increased expression level of Cdk5 induced by HG may be associated with podocyte injury in diabetes (Fig. 3B-F, Supplementary Fig. S2). 


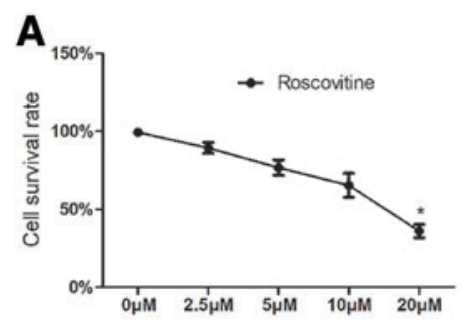

$\mathbf{F}$

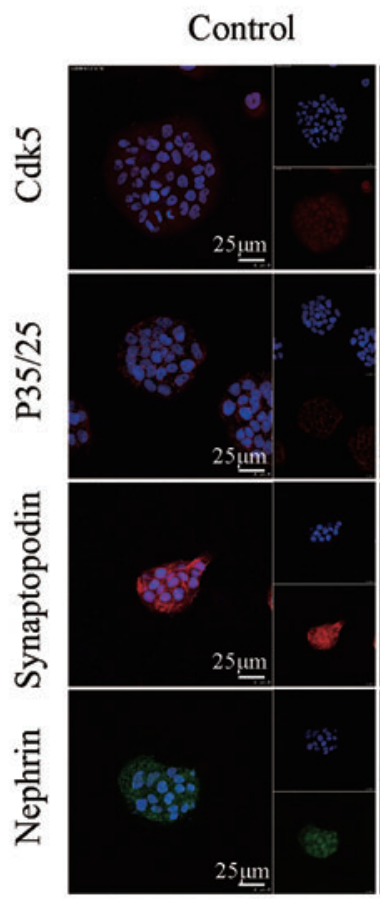

B

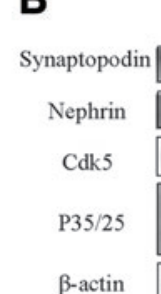

HG

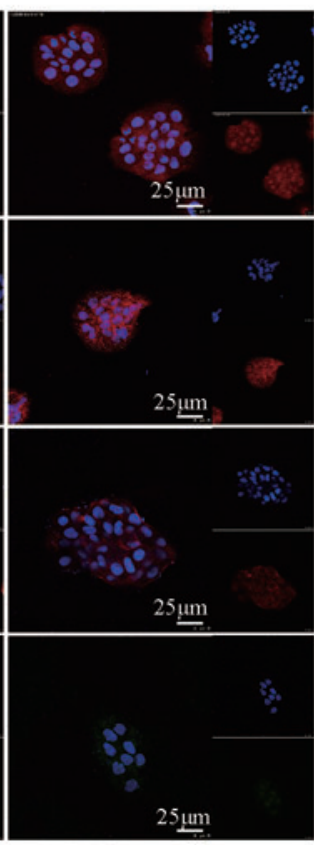

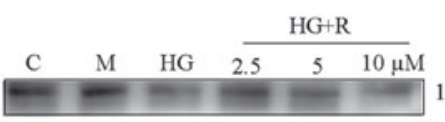

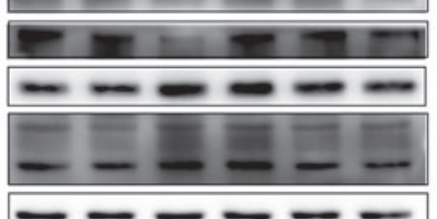

$--\infty-12 \mathrm{kD}$
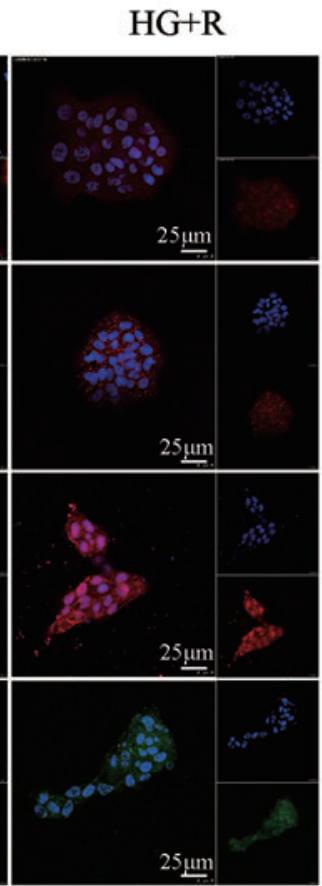
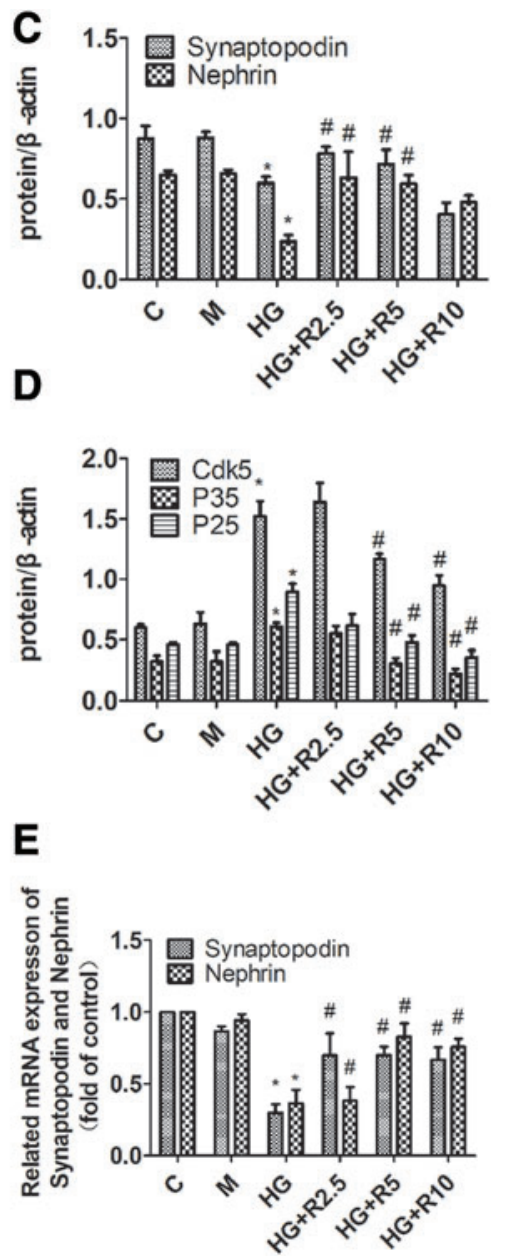

FIG. 3. Roscovitine, the inhibitor of Cdk5, attenuated podocyte injury induced by $\mathrm{HG}$ at $24 \mathrm{~h}$. (A) The cytotoxicity detection of roscovitine by CCK8. ${ }^{*} p<0.05$ compared with the $0 \mu M$ group. (B) Protein representative Cdk5, P35/25, synaptopodin, and nephrin in HPCs. (C, D) Protein levels of Cdk5, P35/25, synaptopodin, and nephrin. (E) The mRNA levels of synaptopodin and nephrin were analyzed by RT-qPCR. (F) The expression of Cdk5, P35/25, synaptopodin, and nephrin by immunofluorescence. DAPI is a nucleus dye. Scale bar: $25 \mu \mathrm{m}$. The data represent the mean \pm SD $(n=3)$. C, control; M, mannitol; R, roscovitine. ${ }^{*} p<0.05$ compared with the control group, ${ }^{\#} p<0.05$ compared with the HG group. Please refer to Supplementary Figure S2 for raw Western blot records shown in the figure. RT-qPCR, quantitative real-time polymerase chain reaction. Color images are available online.

Increasing evidence indicates that the dysfunction of mitochondrial plays many roles in DN pathogenesis $(25$, 26). To assess the role of Cdk5 in podocyte mitochondrial dysfunction, we first detected the expression of dynamicrelated protein 1 (Drp1) and mitofusin 1 (Mfn1). The upregulation of Drp1, a mitochondrial fission protein, was observed in HG-treated podocytes, and especially, the phosphorylation level of Drp1 increased significantly (Fig. 4A, B, Supplementary Fig. S2). However, Mfn1, a mitochondrial fusion protein, was significantly decreased in podocytes (Fig. 4A, B, Supplementary Fig. S2). Furthermore, we detected an upregulation in the expression of mitochondrial damage-related protein, Cyto C, in HGtreated podocytes at $24 \mathrm{~h}$ (Fig. 4A, B, Supplementary Fig. S2).

FIG. 4. Cdk5 suppression alleviated the HG-induced mitochondrial dysfunction at 24 h. (A) Protein representative Drp1, p-Drp1, Mfn1, SOD2, and CytoC in HPCs. (B) Protein levels of Drp1, p-Drp1, Mfn1, and CytoC. (C, D) The MitoTrackerTM Deep Red FM (50 nM) was visualized under confocal microscopy and it was quantified. Scale bar: $10 \mu \mathrm{m} .(\mathbf{E}, \mathbf{F}) \mathrm{The}_{\mathrm{MitoSOX}}^{\mathrm{TM}}$ Red was visualized under confocal microscopy and it was quantified. Scale bar: $10 \mu \mathrm{m} .(\mathbf{G}, \mathbf{H})$ ROS generation was measured by flow cytometry and was quantified following DCFH-DA $(10 \mu M)$ staining. (I, J) The mitochondrial membrane potential in HPCs was measured by JC-1 staining using confocal microscopy. Scale bar: $10 \mu \mathrm{m}$.(K, L) The mitochondrial membrane potential was measured by flow cytometry and was quantified following JC-1 (100 $\mu \mathrm{m})$ staining. (M, N) Representative micrographs showing SA- $\beta$-gal staining in HPCs. Scale bar: $50 \mu \mathrm{m}$. (O) ATP concentrations were determined using an ATP determination kit. The data represent the mean $\pm \mathrm{SD}(n=3)$. C, control; $\mathrm{M}$, mannitol; R, roscovitine; $\mathrm{p}-$, phospho-. ${ }^{*} p<0.05$ compared with the control group, ${ }^{*} p<0.05$ compared with the HG group. Please refer to Supplementary Figure S2 for raw Western blot records shown in the figure. ROS, reactive oxygen species. Color images are available online. 

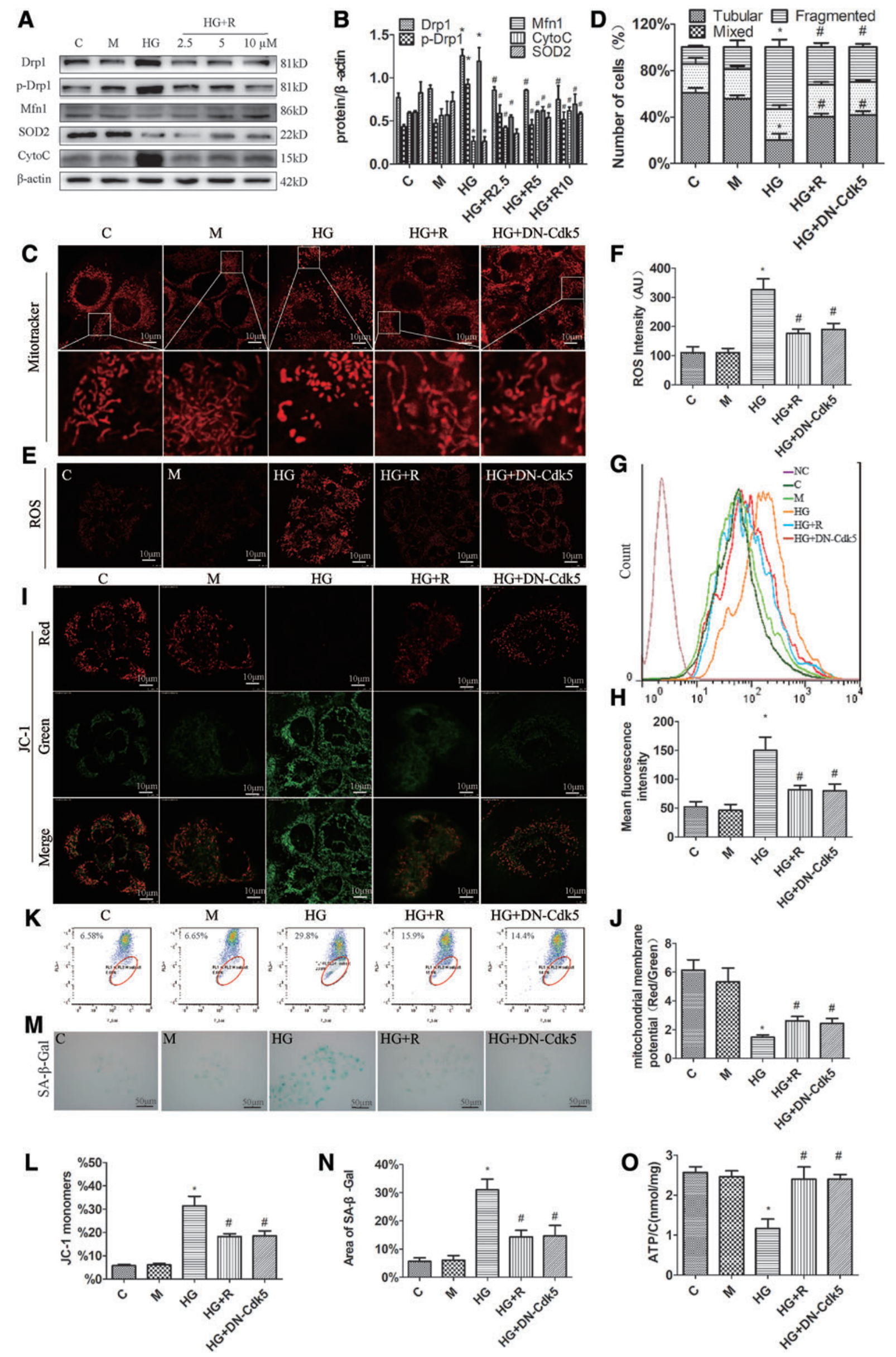
The mitochondrial morphology in human podocyte was examined by MitoTracker Red staining. In physiological conditions, mitochondria appeared thread-like in human podocyte. Treatment with HG markedly affected mitochondrial shape; elongated mitochondria became much shorter and rounder in morphology under these conditions. In contrast, pretreatment with $\mathrm{Cdk} 5$ inhibitor, roscovitine, or knockdown of Cdk5 with DN-Cdk5, significantly prevented the HG-induced changes in mitochondrial morphology (Fig. 4C, D). Using DCFH-DA assay, we demonstrated that the ROS level increased after HG treatment. Simultaneously, SOD2, an ROS scavenger, was decreased (Fig. 4A, B, E-H). Mitochondrial transmembrane potential $(\triangle \Psi \mathrm{M})$ analysis confirmed that HG caused accumulation of JC-1 monomers, indicating the occurrence of decreased $\Delta \Psi \mathrm{M}$ (Fig. 4I-L). Adenosine triphosphate (ATP) concentrations were also decreased after $\mathrm{HG}$ exposure (Fig. 4O). In addition, $\mathrm{HG}$ increased HPC senescence (Fig. 4M, N). Treatment with roscovitine or knockdown $\mathrm{Cdk} 5$ expression reversed all these changes in mitochondria caused by HG. Taken together, these results provide evidence that the over- activation of Cdk5 induced by hyperglycemia is an important reason for podocyte mitochondrial dysfunction and injury in diabetes.

\section{Phospho-Sirt1 is upregulated in the glomeruli of patients with DN and human podocytes}

Sirt1 is an NAD-dependent deacetylase that has a protective effect in the maintenance of mitochondrial function (3, 46). Phosphorylation of Sirt1 has negative effects on cellular functions (11), so we assessed levels of phosphor-Sirt1 in diabetic glomeruli. Positive staining of p-Sirt1 was located in the nucleus of DN glomerular cells (Fig. 5A). The expression level of p-Sirt1 in DN glomeruli was significantly higher than that in control glomerulus (Fig. 5B). In contrast to p-Sirt1, the expression level of Sirt1 in DN glomeruli was significantly lower than that in control glomerulus (Fig. 5A, C). Western blotting results indicated that Sirt1 expression was significantly downregulated after HG treatment (Fig. 5D, F, Supplementary Figure S3), where p-Sirt1 level was increased $12 \mathrm{~h}$ after $\mathrm{HG}$ treatment and reached its highest levels at 24 and $48 \mathrm{~h}$ (Fig. 5D, E, Supplementary Figure S3).
A
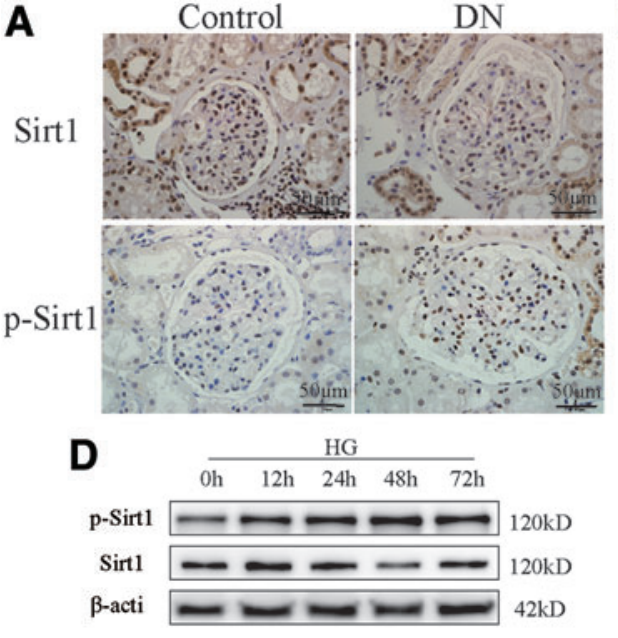
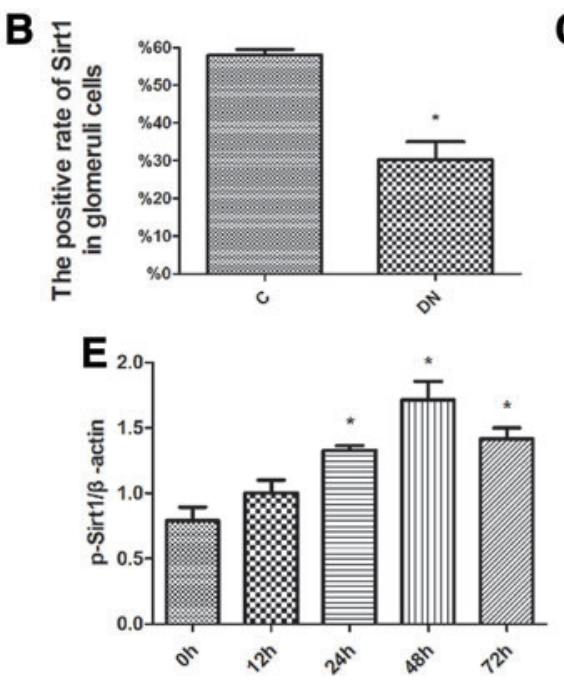
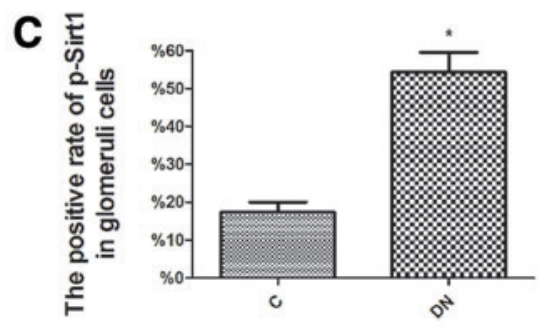

$\mathbf{F}$

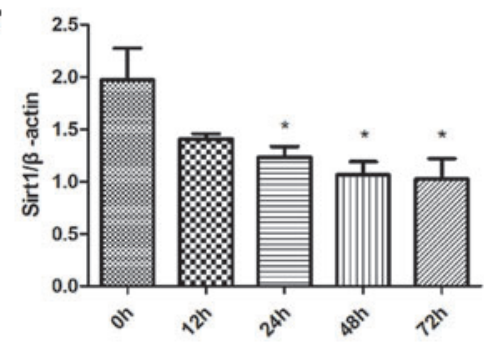

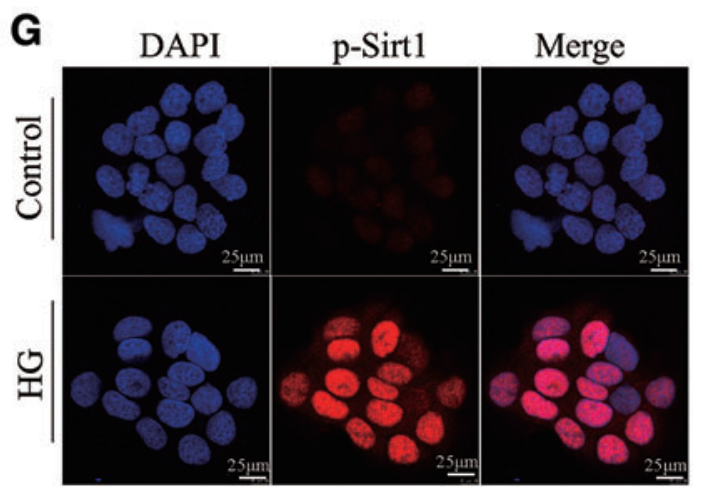

H

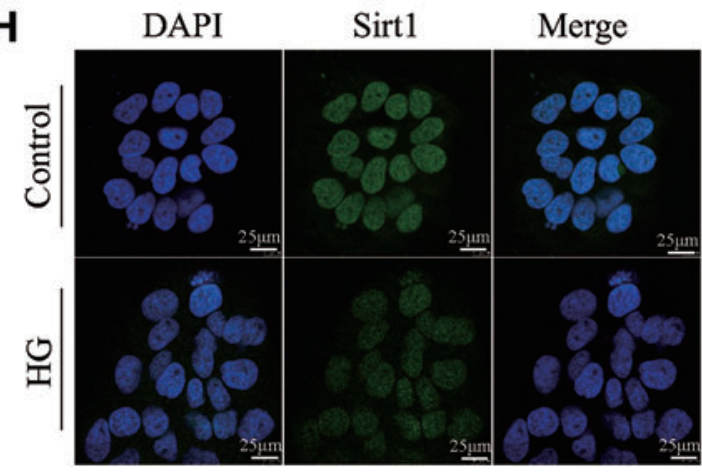

FIG. 5. HG upregulated phosphor-Sirt1 in the glomeruli of DN patients and human podocytes. (A) Representative immunohistochemical staining for $\mathrm{p}$-Sirt1 and Sirt1 in the glomerulus. Scale bars: $50 \mu \mathrm{m}$. Magnification: $400 \times$. (B, C) Quantification of p-Sirt1 and Sirt1 positive rate in the glomerulus. $(p<0.05, n=19)$ (D) Protein representative Sirt1 and p-Sirt1 in HPCs induced by $30 \mathrm{~m} M$ glucose for different time points. (E, F) Protein levels of p-Sirt1 and Sirt1. (G, H) The expression of p-Sirt1 and Sirt1 in HG-induced HPCs by immunofluorescence at $24 \mathrm{~h}$. DAPI is a nucleus dye. Scale bar: $25 \mu \mathrm{m}$. The data represent the mean \pm SD $(n=3)$. p-, phospho-. ${ }^{*} p<0.05$ compared with the $0 \mathrm{~h}$ group. Please refer to Supplementary Figure S3 for raw Western blot records shown in the figure. Color images are available online. 
Immunofluorescence analysis indicated that Sirt 1 and p-Sirt1 were all expressed in the nuclei in podocytes. Following HG treatment, p-Sirt1 markedly expressed HG cells than in control cells, but Sirt1 expression decreased (Fig. 5G, H).

We used HG to stimulate HPCs at $0,1,3,6,12,24,48$, and $72 \mathrm{~h}$ and found that although Mcl-1 expression decreased at 1 , 3 , and $6 \mathrm{~h}$, it remained unchanged at $24 \mathrm{~h}$ as it was at $0 \mathrm{~h}$. At the same time, we treated HPCs with resveratrol (RSV), a Sirt1 activator, or EX527, a Sirt1 inhibitor, and found that Mcl-1 expression remained the same at $24 \mathrm{~h}$. In addition, we treated HPCs with Marinopyrrole A, a Mcl-1 inhibitor, and found that the expression of Sirt1 and p-Sirt1 remained unchanged. We speculate that HG promotes mitochondrial dysfunction through Cdk5-Sirt1 at $24 \mathrm{~h}$, which is not associated with Mcl-1 (Supplementary Fig. S4A-J).

\section{Downregulated phospho-Sirt1 alleviates the mitochondrial dysfunction and podocyte injury induced by HG}

Studies have shown that $\mathrm{Cdk} 5$ regulates the phosphorylation of Sirt1 at S47 site (4). Thus, we investigated whether Cdk5 mediated the mitochondrial dysfunction of podocyte by phosphorylating Sirt1 at the same site. To further investigate the role of Sirt1 phosphorylation on podocyte mitochondrial dysfunction, we constructed a mutant plasmid of Sirt1 at S47, in which the serine was replaced with an alanine (Flag-Sirt1S47A). The mutant Sirt1-S47A mimicked the nonphosphorylated form (Fig. 6A, Supplementary Fig. S5).

As shown in Figure 6 and Supplementary Figure S5, compared with the group transfected with pcDNA3.0, the downregulation of synaptopodin and nephrin induced by $24 \mathrm{~h}$ HG was significantly reversed by transfected mutant Sirt1 (S47A) (Fig. 6B-D). Drp1 expression was markedly increased with HG treatment, accompanied with p-Drp1 upregulation in transfected control cells (Fig. 6B, E, F); CytoC levels were also increased in these cells (Fig. 6B, I). In contrast, Mfn1 and SOD2 levels were significantly decreased (Fig. 6B, G, H). The mutation of Sirt1 with mutant S47A caused opposite effects on the expression of these proteins (Fig. 6B-I). MitoTracker Red staining showed that HGinduced mitochondrial swelling was alleviated in the presence of mutant Sirt1 (S47A) (Fig. 6J and Supplementary Fig. S6A). Furthermore, mutating Sirt1 (S47A) to prevent its phosphorylation also reduced ROS generation (Fig. 6K-M and Supplementary Fig. S6B) and increased $\triangle \Psi \mathrm{M}$ (Fig. $6 \mathrm{~N}$, $\mathrm{O}$ and Supplementary Fig. S6C, D), ATP levels (Fig. 6Q), and senescence levels (Fig. 6P and Supplementary Fig. S6E).

\section{S47 phosphorylation of Sirt1 is mediated by Cdk5 in podocytes after HG treatment}

We used immunofluorescence to investigate the interaction between Cdk5 and Sirt1 in human tissues. In Figure 7A, compared with control cells, Cdk5 expression was increased in the glomeruli of DN, whereas Sirt1 was less expressed. As shown in Figure 7B, p-Sirt1 expression significantly increased, accompanied with the upregulation of Cdk5. The results also demonstrated that p-Sirt 1 colocalized with Cdk5 in glomerular cells. Following HG treatment in HPCs, p-Sirt1 increased and colocalized with Cdk5 in podocyte nucleus. Contrast to p-Sirt1, the Sirt1 downregulated and colocalized with $\mathrm{Cdk} 5$ in podocyte nucleus (Fig. 7C, D). We performed co-immunoprecipitation
(Co-IP) experiments to verify the interaction of Cdk5 and Sirt1 in HPCs. As shown in Figure 7E and Supplementary Figure $\mathrm{S} 7$ and F, Cdk5 was increased by $24 \mathrm{~h} \mathrm{HG}$ in Sirt 1 IP, whereas Sirt1 and p-Sirt1 were also increased in Cdk5 IP.

To explore the effect of Cdk5 on Sirt1 phosphorylation, we first transfected HPCs with WT-Cdk5, the wild-type plasmid of Cdk5. The results showed that WT-Cdk5 plasmid increased the expression and kinase activity of $\mathrm{Cdk} 5$ (Fig. 8A, D, E) and upregulated the expression level of p-Sirt1 in HPCs (Fig. 8H, I). To further detect the kinase activity of Cdk5 on p-Sirt1 expression, p25 plasmid, which could overactivate Cdk5, was transfected into HPCs (Fig. 8B, C). Aberrant activation of Cdk5 via overexpressed p25 also increased the phosphorylation level of Sirt1 in HPCs (Fig. 8F, G). To determine the Cdk5 phosphorylation site on Sirt1, the mutant Sirt1 plasmid (Sirt1-S47A) was cotransfected with WT-Cdk5 or p25 plasmid. We demonstrated that Sirt1-S47A decreased the Cdk5-induced phosphorylation of Sirt1 in HPCs (Fig. 8F-I). Furthermore, the kinase activity and mRNA expression of Cdk5 were inhibited by roscovitine and DN-Cdk5, respectively. The results showed that inhibition of Cdk5, whether its kinase activity or mRNA expression, significantly reduced p-Sirt1 expression induced by HG (Fig. 8J, K).

In normal podocytes transfected with WT-Cdk5, we observed a marked decrease in nephrin and synaptopodin expression, which was reversed by cotransfection with Sirt1S47A. Immunoblot analyses revealed that the upregulation of Drp1, p-Drp1, and Cyto C, and the downregulation of Mfn1 and SOD2 induced by WT-Cdk5 were also reversed by Sirt1S47A (Fig. 8L, M, Supplementary Fig. S8). In addition, mitochondrial swelling, increased ROS, increased senescence, decreased ATP, and the loss of mitochondrial voltage potential induced by $\mathrm{Cdk} 5$ overexpression were also attenuated by Sirt1-S47A (Fig. 8N-U and Supplementary Fig. S9). As shown in Figure $8 \mathrm{~V}$ and $\mathrm{W}$, we verified that ROS was involved in Cdk5-mediated podocyte injury. Thus, these results suggest that Cdk5 promotes Sirt1 phosphorylation at S47, leading to mitochondrial dysfunction and podocyte damage.

\section{Cdk5 downregulation by shRNA lentivirus attenuates podocyte injury in diabetic mice}

Our finding demonstrated that Cdk5 inhibition might be a valuable therapeutic approach for DN. We used lentivirus vectors to deliver Cdk5 shRNA to STZ-induced type 1 diabetic mice or type $2 \mathrm{db} / \mathrm{db}$ mice. A significant reduction in the glomerular expression of $\mathrm{Cdk} 5$ was detected in the mice treated with lentivirus-Cdk5 shRNA, with subsequent significant recovery of synaptopodin and nephrin expression (Fig. 9E G and Supplementary Figs. S10E, F and S11). Increased expression of Sirt1 and decreased p-Sirt1 were also observed in lentivirus-Cdk5 shRNA-infected mice (Fig. 9F, G and Supplementary Fig. S10E, $\mathrm{G}, \mathrm{H})$. To evaluate the effects of Cdk5 shRNA on mitochondrial dynamics, the mitochondrial fission and fusion proteins were also assessed. Drp1 and p-Drp1 levels were downregulated and Mfn1 was upregulated (Supplementary Fig. S11); and the abnormal increase in CytoC expression was restored by lentivirus-Cdk5 shRNA treatment in diabetic mice (Supplementary Fig. S11).

Furthermore, transmission electron microscopy (TEM) results confirmed the alleviation of podocyte FPs effacement, glomerular basement membrane thickening, and mitochondrial swelling in lentivirus-Cdk5 shRNA-treated mice compared with diabetic mice (Fig. 9B-D and Supplementary 


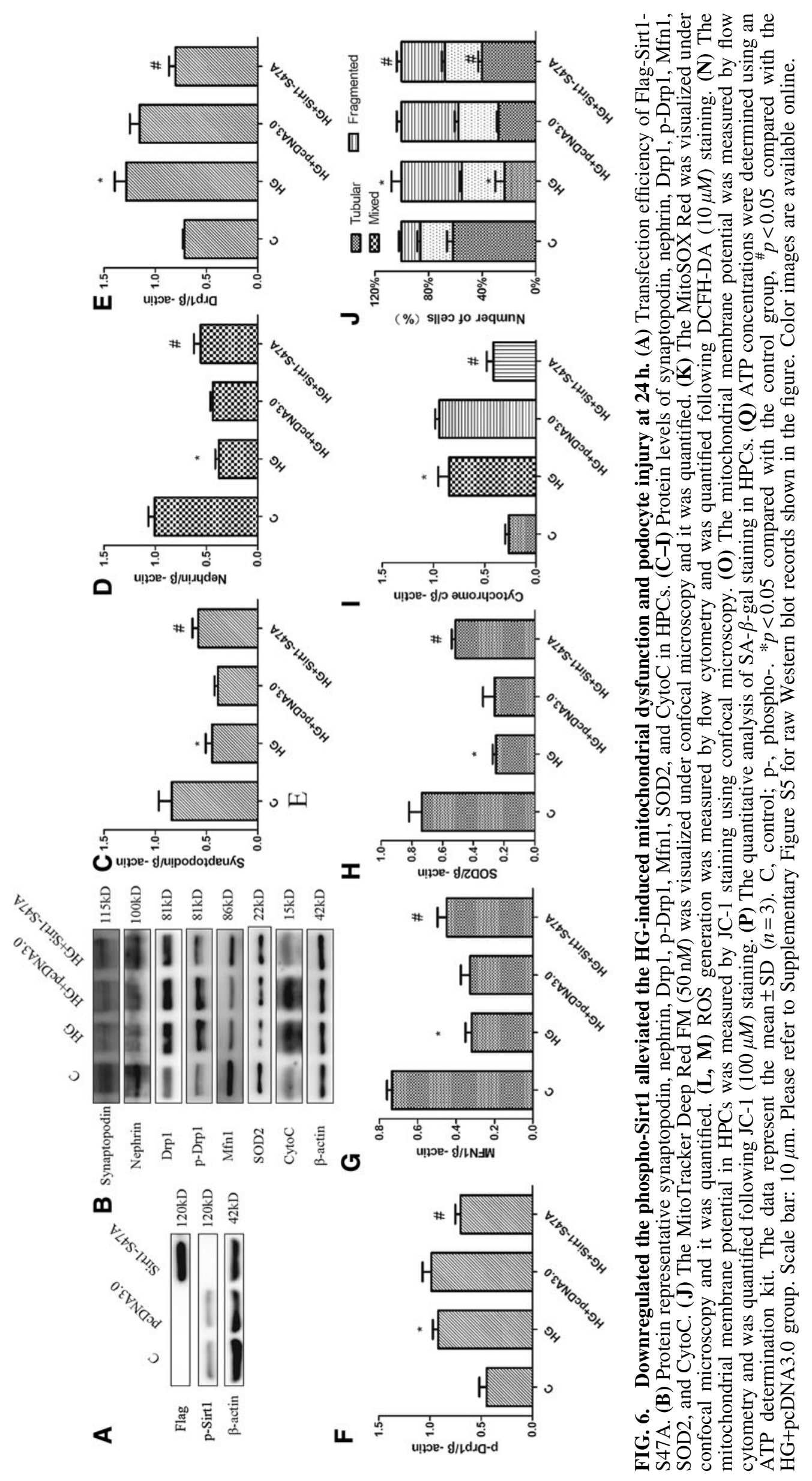



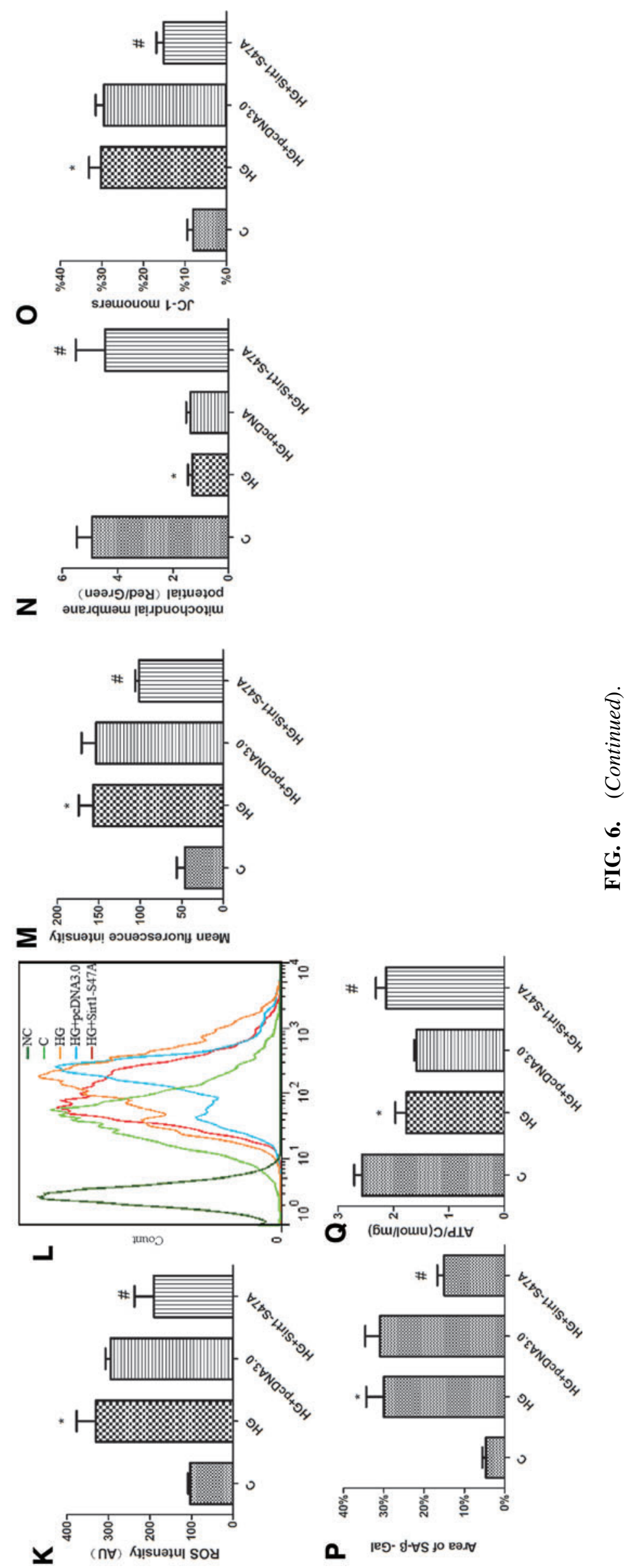

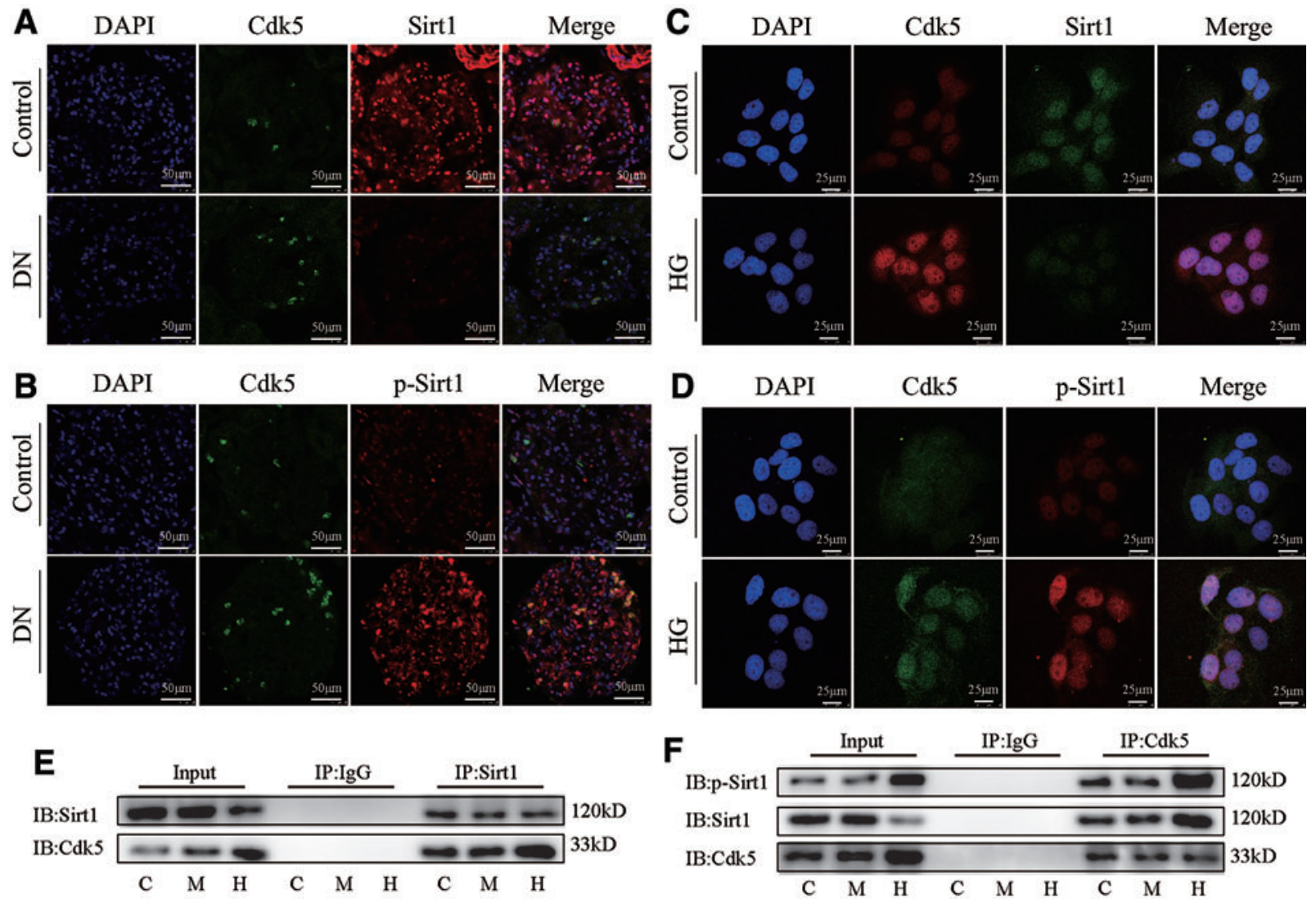

FIG. 7. The interaction between Cdk5 and Sirt1. (A, B) Colocalization of Cdk5 with Sirt1 and p-Sirt1 in DN kidney tissues by immunofluorescence. DAPI is a nucleus dye. Scale bars: $50 \mu \mathrm{m}$. Magnification: $400 \times$. (C, D) Colocalization of Cdk5 with Sirt1 and p-Sirt1 in the HPCs treated with HG $(30 \mathrm{mM})$ by immunofluorescence at $24 \mathrm{~h}$. DAPI is a nucleus dye. Scale bars: $25 \mu \mathrm{m}$. (E) Co-IP analysis demonstrated that Cdk5 protein in Sirt1 precipitates was enhanced by HG $(30 \mathrm{~m} M)$ at $24 \mathrm{~h}$. (F) Co-IP analysis demonstrated that Sirt1 and p-Sirt1 protein in Cdk5 precipitates were enhanced by HG (30 m $M$ ) at 24 h. C, control; p-, phospho-. Please refer to Supplementary Figure S7 for raw Western blot records shown in the figure. Color images are available online.

Fig. S10B-D). Finally, analyses of biochemical parameters showed that urinary albumin, serum creatinine, and blood urea nitrogen (BUN), which were notably increased in diabetic mice, were decreased in lentivirus-Cdk5 shRNAtreated mice (Fig. 9A and Supplementary Fig. S10A).

\section{Discussion}

Mitochondria are the primary organelles of cell energy production, and their dysfunction is an important reason for podocyte injury in DN $(40,41,49)$. The mechanism of mitochondrial dysfunction in podocytes in diabetes is not fully understood. In the current study, we demonstrated that Cdk5mediated Sirt1 phosphorylation contributes to mitochondrial dysfunction in podocytes in diabetic mice and cell models.

$\mathrm{Cdk} 5$ is a proline-directed serine/threonine protein kinase that is expressed in podocytes and plays an important role in the maintenance of their normal function (44). Knockdown of Cdk5, or its activator, p35, results in podocytes apoptosis (7, $30,57)$. Although many studies have demonstrated that deregulated $\mathrm{Cdk} 5$ is implicated in neurodegenerative diseases, such as $\mathrm{AD}$ and $\mathrm{PD}$, the role of aberrant $\mathrm{Cdk} 5$ in podocyte injury has not been thoroughly investigated $(19,54)$. In this study, we found an upregulation in the expression of Cdk5 in the podocytes of diabetic mice and patients with DN, which negatively correlated with nephrin and synaptopodin expressions. The expression of nephrin and synaptopodin was decreased with HG treatment in podocytes and was recovered by $\mathrm{Cdk} 5$ inhibitor roscovitine. The result demonstrated that Cdk5 overactivated by $\mathrm{HG}$ is an important reason for podocyte injury in diabetes.

The kidney is one of the most energy-demanding organs and requires a lot of mitochondria to provided sufficient energy to maintain its normal function (13). Mitochondrial homeostasis imbalance and energetics damage contribute to the progression of kidney diseases, such as DN (51). Hyperglycemia is the initiating factor and causes of mitochondrial structural and functional damages in the progression of DN, especially in podocyte injury (21). The present study confirmed that HG treatment dramatically damaged the mitochondria in podocytes. We observed significant mitochondrial swelling and fragmentation following a HG treatment. Mitochondria are highly dynamic organelles and constantly undergoing fission and fusion to maintain the mitochondrial homeostasis in physiological conditions (16). In diabetic conditions, the mitochondrial fission in podocytes significantly increased (32). Cdk5 mediates mitochondrial fission in neurodegenerative diseases (18). The current study showed that inhibition of $\mathrm{Cdk} 5$ by roscovitine or shRNA significantly decreased the number of fragmented 


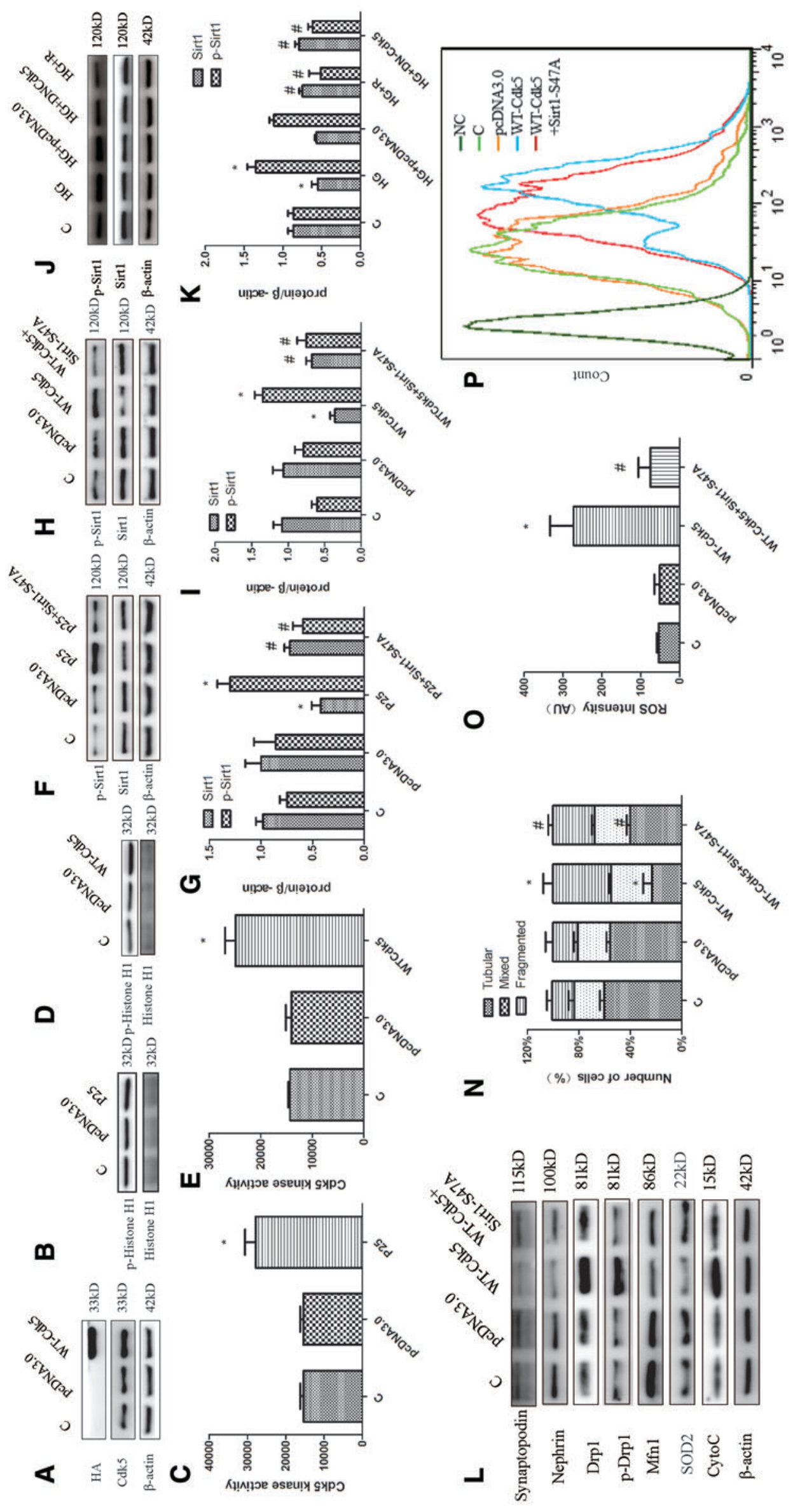

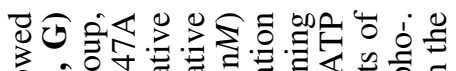

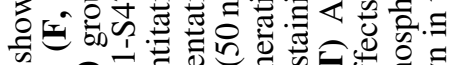

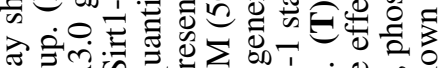

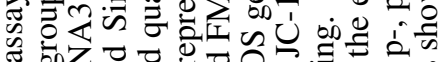
to

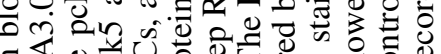

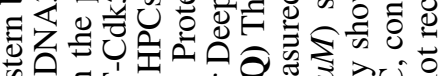

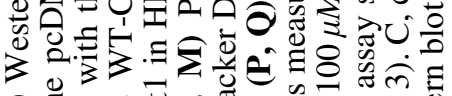

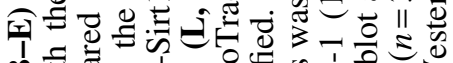

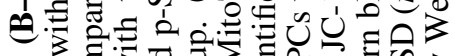

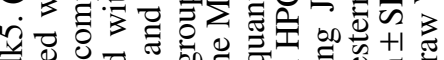

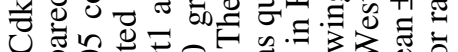

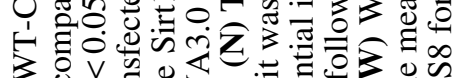
3 ov 0

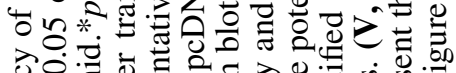

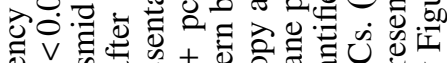

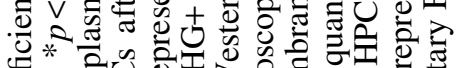

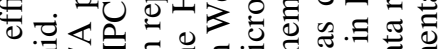

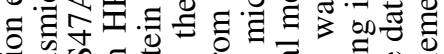

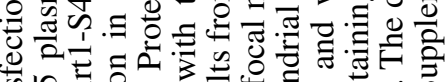

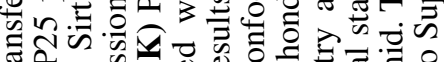

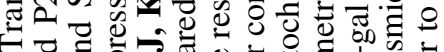

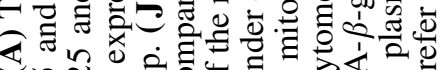

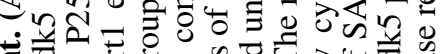

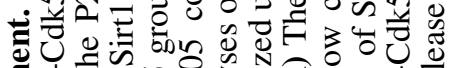
赔 का

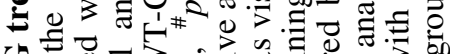

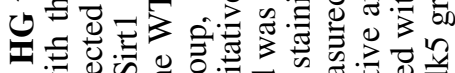

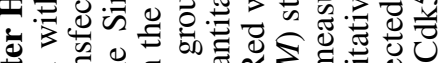

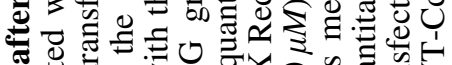

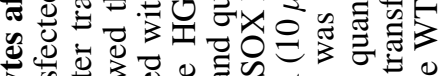

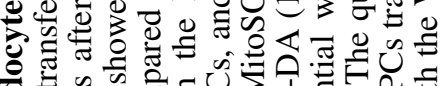

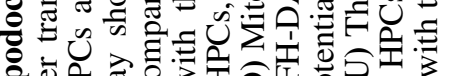

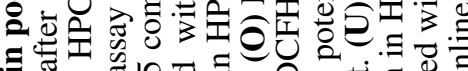

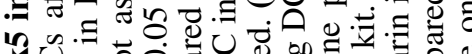

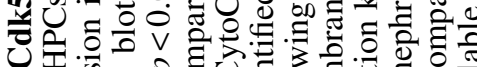
I. 호예․

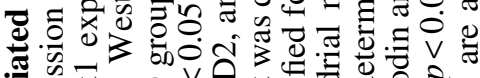

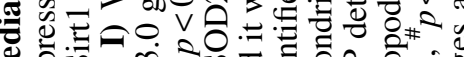
눙

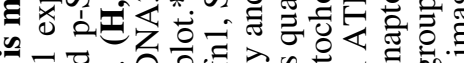

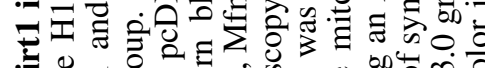
क o

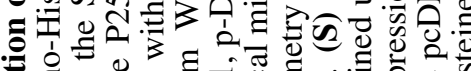

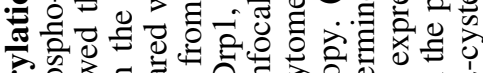

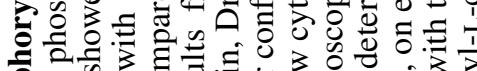
के

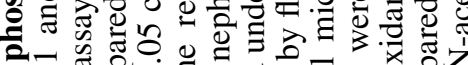

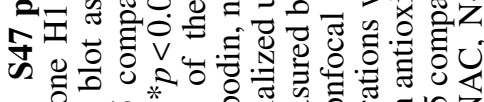

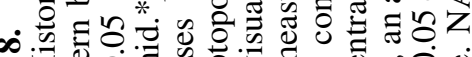

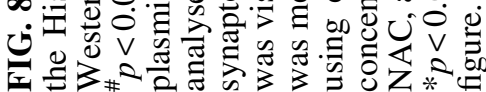



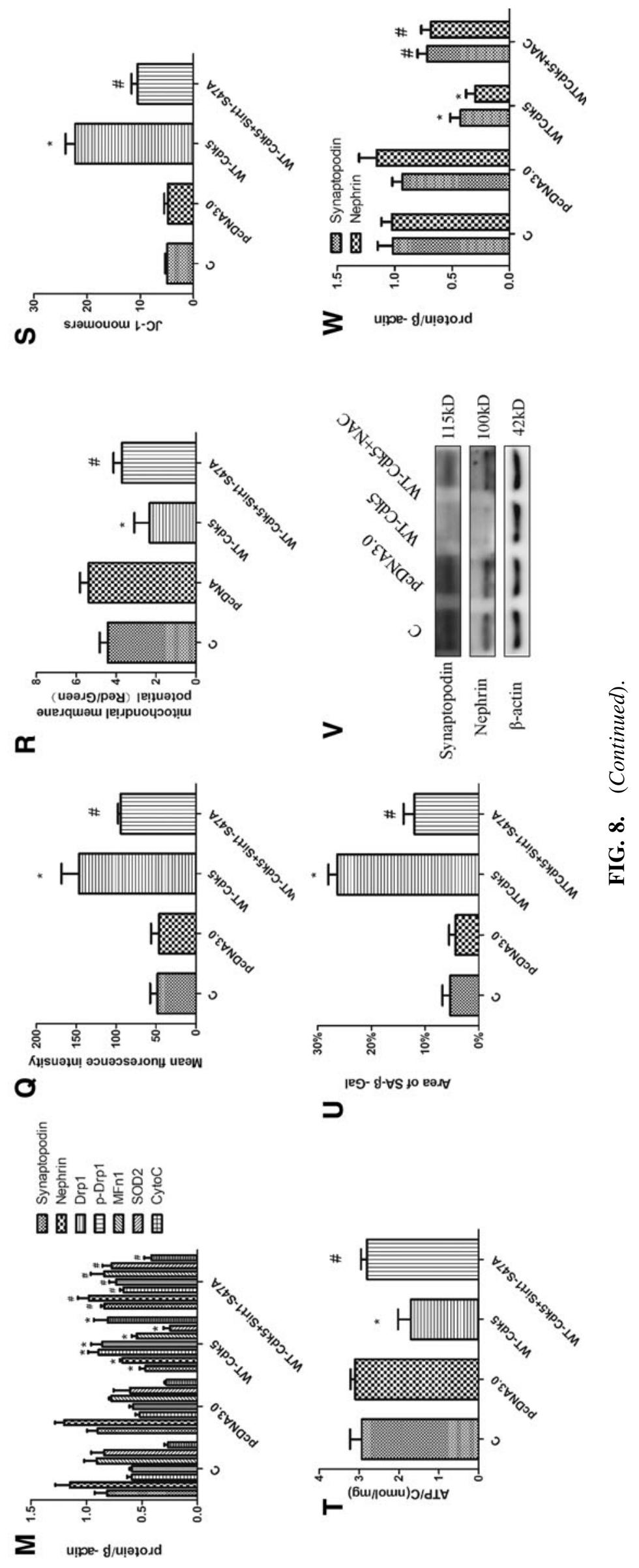

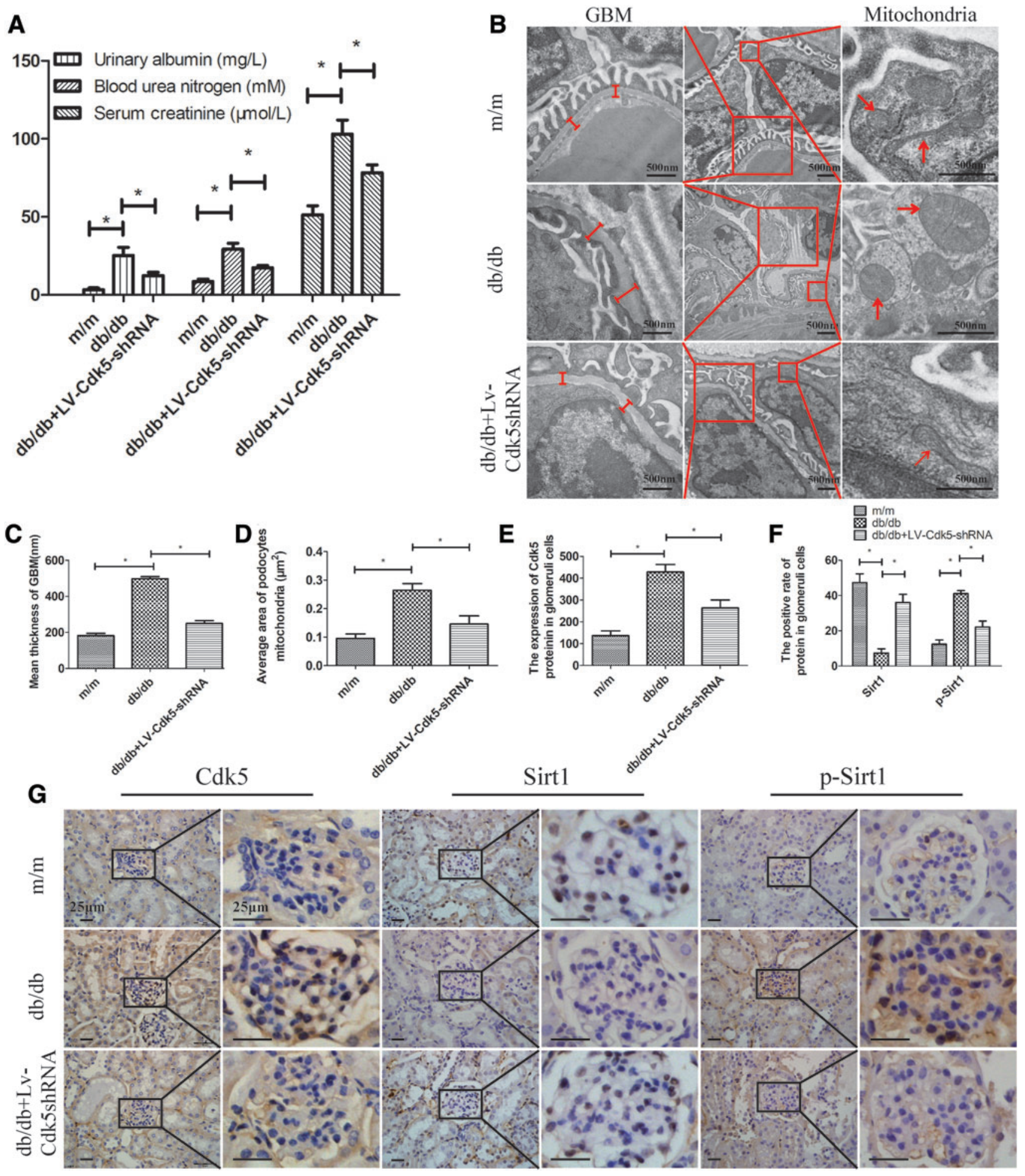

FIG. 9. Lentivirus-Cdk5 shRNA attenuated podocyte injury in diabetic mice. (A) Urinary albumin (mg/L), serum creatinine $(\mu M)$, and blood urea nitrogen $(\mathrm{m} M)$ of $\mathrm{db} / \mathrm{db}$ mice.(B-D) Transmission electron microscopy assay showed the ultrastructure of the podocytes in renal cortex of $\mathrm{db} / \mathrm{db}$ mice. Red lines referred to the thickness of the GBM, and red arrows referred to mitochondria of podocytes. Scale bar: $500 \mathrm{~nm}$. Magnification: $20,000 \times$. The data represent the mean \pm SD $(n=5) .{ }^{*} p<0.05$ (E-G) Immunohistochemical staining of Cdk5, Sirt1, and p-Sirt1 in the glomerulus from db/db mice. Scale bars: $50 \mu \mathrm{m}$. Magnification: $400 \times$. The data represent the mean $\pm \mathrm{SD}(n=5)$. GBM, glomerular basement membrane; p-, phospho-. Color images are available online. 
mitochondria and elongated it. Drp1 and Mfn1 are the major proteins that control mitochondrial fission and fusion. Wang et al. showed that ROCK1 promotes mitochondrial fission by recruiting Drp1 to the mitochondria in diabetic mice (47). Phosphorylation of Drp1 also promotes mitochondrial fission and DN progression $(17,50)$. In our study, the expression of Drp1 and phosphorylated Drp1 was significantly increased and that of Mfn1 decreased following a HG treatment, whereas these changes were reversed by Cdk5 suppression. Hyperglycemia promotes ROS generation, which caused the decrease in ATP production, the release of Cyto $\mathrm{C}$, and the dysfunction of mitochondria $(22,35)$. Inhibition of Cdk5 also effectively reduced ROS production and CytoC release in HG-treated podocyte and rescued ATP generation and $\triangle \Psi \mathrm{M}$.

Sirt1 is an $\mathrm{NAD}^{+}$-dependent deacetylase that belongs to the sirtuin family (34). Sirt1 participates in mitochondrial energy metabolism through deacetylation of PGC- $1 \alpha$ (9). There is a growing literature describing the activation or upregulation of Sirt1 protecting DN from inflammation, apoptosis, and fibrosis $(14,43,48)$. It has been shown that Sirt1 protects podocyte against the damage of hyperglycemia in diabetes mellitus. Podocyte-specific Sirt1 deletion leads to increased urinary protein level and the aggravation of renal injury $(20,29)$. Our study also showed the decreased expression of Sirt1 in podocytes following a HG treatment.

Sirt1 activators, such as RSV and other synthetic drugs, have not been shown definitively to have beneficial effects on DN in clinical trials. There are differences in the effects of Sirt1 activators between clinical and preclinical studies. Posttranslational modification is an important method to regulate the activity of Sirt1. Phosphorylation regulation can significantly reduce Sirt1 activities (12). We surmise that intervention from the perspective of post-translational modification may enhance Sirtl activity and its therapeutic effects on DN. During the progression of podocyte injury in vivo and in vitro, the phosphorylation of Sirt1 was significantly upregulated at S47, leading to mitochondrial dysfunction. Inhibition of Sirt1 at $\mathrm{S} 47$ by mutating the single amino acid ameliorates the podocyte injury, mitochondrial dynamic and mitochondrial dysfunction in HG-treated podocyte. This demonstrated that increased phosphorylation of Sirt1 caused mitochondrial dysfunction and leads to podocyte damage.

Overactivated Cdk5 leading to more phosphorylation of its target substrates is an important underlying factor for several diseases, such as $\mathrm{AD}$ and $\mathrm{PD}(36,40,56)$. Also, Cdk5 was reported to phosphorylate Sirt1 and inhibit its antisenescent and anti-inflammatory activities (4). The present study confirmed that overactivated $\mathrm{Cdk} 5$ upregulated the level of phosphorylated Sirt1 at S47. Suppression of Cdk5 downregulated the Sirt1 phosphorylation level, and the nonphosphorylation mutation form S47A abolished the mitochondrial dysfunction and podocyte injury induced by WT-Cdk5 plasmid. These results indicate that $\mathrm{Cdk} 5$ overactivation promotes Sirt1 phosphorylation is an important reason for mitochondrial dysfunction and podocyte injury in DN.

In in vitro study, knockdown of Cdk5 expression by lentivirus-Cdk5 shRNA significantly attenuated podocyte injury. From our points of view, Cdk5 interference may enhance the therapeutic effect of RSV and other Sirt1 activators by increasing Sirt1 activity through post-translational modifications. Thus, Cdk5-Sirt1 signaling pathway may be a valuable therapeutic target for DN.

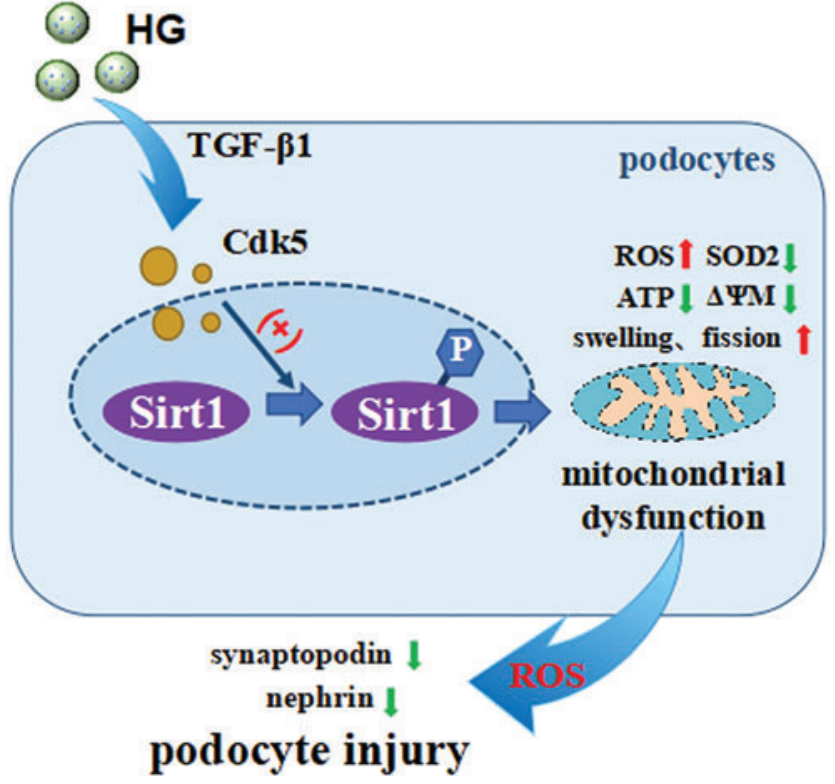

FIG. 10. Mechanistic depiction of the role of Cdk5 in podocyte mitochondrial dysfunction. Our study reveals foremost that Cdk5 as critical kinase by promoting Sirt1 phosphorylation at S47 participates in mitochondrial dysfunction and podocyte injury, which leads to the progression of diabetic nephropathy. Color images are available online.

The current study firmly established that Cdk5, as a critical kinase that promotes Sirt1 phosphorylation at S47, participates in mitochondrial dysfunction and podocyte injury, which leads to the progression of DN. Inhibition of Cdk5 activities may be a potential target to reverse podocyte injury and reduce the progression of DN (Fig. 10).

\section{Materials and Methods}

\section{Reagents}

McCoy's 5A (Modified) Medium and fetal calf serum were purchased from Gibco (Carlsbad, CA). D-glucose was obtained from Solarbio (Beijing, China). FuGENE HD Transfection Reagent was purchased from Promega (Madison, WI). Antibodies against Cdk5 (ab40473), Drp1 (ab184247), nephrin (ab58596), and Sirt1 (ab110304) were purchased from Abcam (Cambridge, United Kingdom). Antibodies for $\beta$-actin (20536-1-AP), Mfn1 (13798-1-AP), and synaptopodin (21064-1-AP) were obtained from Proteintech Group (Wuhan, Hubei, China). Antibodies for p-Sirt1 (2314), p35/25 (2680), and p-Drp1 (3455) were purchased from Cell Signaling Technology (Beverly, MA). Antibodies against SOD2 (EM1717-32) and Cdk5 (SR39-01) were purchased from HuaBio Technology (Hangzhou, Zhejiang, China). WT-Cdk5, DN-Cdk5, and Sirt1 plasmids were obtained from Addgene (Watertown, MA). MitoTracker ${ }^{\mathrm{TM}}$ Deep Red FM and MitoSOX ${ }^{\mathrm{TM}}$ Red were purchased from Thermo Fisher (Waltham, MA). DCFH-DA was purchased from Sigma (St. Louis, MO). JC-1 was purchased from Cayman Chemical (Ann Arbor, MI). Roscovitine and STZ were obtained from Sigma (Shanghai, China). Enhanced ATP Assay Kit was obtained from Beyotime (Shanghai, China). Biochemical detection kits for $24 \mathrm{~h}$ urinary protein, BUN, and serum 
creatinine (Scr) were purchased from Nanjing Jiancheng Bioengineering Institute (Nanjing, Jiangsu, China). Mut Express II Fast Mutagenesis Kit V2 was obtained from Vazyme (Nanjing, Jiangsu, China). Senescence $\beta$-Galactosidase Staining Kit (C0602) was purchased from Beyotime (Shanghai, China). Takara (Shiga, Japan) was the source of SYBR Premix Ex Taq II.

\section{Human renal specimens}

Nineteen patients aged 30-55 years diagnosed with DN were recruited from the Inpatient Department of Nephrology at the Second Hospital of Hebei Medical University from 2017 to 2018 . The study protocols concerning human tissues are consistent with the principles of the Declaration of Helsinki and were approved by the Clinical Research Ethics Committee of Hebei Medical University (IRB number: 20190040). Nineteen samples of control kidney tissues were acquired from nephrectomies performed for the treatment of renal lipomyoma and pathologically confirmed normal kidney tissues without history of other autoimmune diseases, DN, hypertension nephropathy, or severe infections. Informed consent was obtained from each patient, and patients' clinical biochemical data were extracted from medical records. Assessment of the histopathology was evaluated in a blinded manner by two experienced pathologists and classified according to the Renal Pathology Society's Pathologic Classification of DN based on glomerular pathology. Kidney tissues were fixed with $4 \%$ formaldehyde for hematoxylin and eosin, periodic acid-Schiff, and immunohistochemical staining.

\section{Animal models}

All animal studies were in accordance with the guidelines from the Care and Use of Laboratory Animals and were approved by the Animal Care and Ethics Committee of Hebei Medical University (approval ID: HebMU 20080026). The mice were housed in an air-conditioned room at $24^{\circ} \mathrm{C}$ with alternating $12 \mathrm{~h}$ cycles of light and dark. All mice were maintained on a standard diet (SPF mice maintain feed; Beijing Keao Xieli Feed Co., Ltd.) and given tap water $a d$ libitum.

\section{STZ mice}

Eight-week-old male CD1 mice (30-40 g) were obtained from Beijing Charles River Laboratory Animal Technology Co. Ltd (Beijing China). After 7 days of adaptation, 24 CD1 mice were randomly divided into 3 groups: control group (8 mice), STZ group (8 mice), and STZ+Lv-Cdk5shRNA group (8 mice). Mice in the STZ group and the STZ+LvCdk5shRNA group were induced by a single intraperitoneal injection of STZ at $150 \mathrm{mg} / \mathrm{kg}$. Control mice received an equal volume of vehicle $(0.1 \mathrm{M}$ citrate buffer, $\mathrm{pH} 4.5)$. Individual animals with blood glucose concentrations of $16.7 \mathrm{~m} M$ for three consecutive days were confirmed as diabetic mice (31).

\section{C57BLKS/J $d b / d b$ mice}

Ten 8-week-old male C57BLKS/J db/db mice (32-36 g) with genetic type 2 diabetes and 5 healthy $\mathrm{m} / \mathrm{m}$ male mice (26$28 \mathrm{~g}$ ) were purchased from GemPharmatech Co., Ltd. (Nanj- ing, China). The $10 \mathrm{db} / \mathrm{db}$ mice were randomly divided into 2 groups: $\mathrm{db} / \mathrm{db}$ group $(n=5)$ and $\mathrm{db} / \mathrm{db}+\mathrm{Lv}-\mathrm{Cdk} 5 \mathrm{shRNA}$ group $(n=5)$. Five control mice served as the $\mathrm{m} / \mathrm{m}$ group.

To verify the protective effect of Cdk5 inhibition on kidney in vivo, eight STZ+Lv-Cdk5shRNA mice and five $\mathrm{db} /$ $\mathrm{db}+\mathrm{Lv}-\mathrm{Cdk} 5 \mathrm{shRNA}$ mice were renally injected with $50 \mu \mathrm{L}$ $1 \times 10^{6}$ infective units of lentivirus-Cdk5 shRNA in both kidneys at 24 weeks. Mice in the control group and the $\mathrm{m} / \mathrm{m}$ group were injected with isometric saline. At the end of 4 weeks after treatment, mice were kept in individual metabolic cages for $24 \mathrm{~h}$ urine collection to measure urine albumin. The mice were anesthetized with $5 \%$ chloral hydrate, and the blood samples were collected for blood glucose, BUN, and serum creatinine (Scr). Then, the mice were sacrificed, and the kidneys were harvested immediately for relevant investigations.

\section{Plasmid and lentivirus construction}

Sirt1 site-directed mutagenesis was performed to generate a construct in which S47 was replaced with alanine (Flag-Sirt1-S47A) (Mut Express II; Vazyme). All steps are according to the manufacturer's instructions. Transient transfection was performed in human podocytes with FuGENE HD transfection reagent, and overexpression was confirmed by Western blotting using the anti-Flag antibody.

For in vivo inhibition, the lentivirus suppressing Cdk5 expression was produced with the short hairpin (sh) RNA sequence 5'-CGGGAGATCTGTCTACTCAAACTCGA GTTTGAG TAGACAGATCTCCCG-3' by Cyagen (Santa Clara, CA, USA). A scrambled shRNA was used as the negative control.

\section{Cell culture and transfection}

The primary HPCs purchased from BeNa Culture Collection (BNCC, Beijing, China) were cultured in McCoy's $5 \mathrm{~A}$ Medium supplemented with $10 \%$ fetal bovine serum, $100 \mathrm{U} / \mathrm{mL}$ penicillin, and $100 \mu \mathrm{g} / \mathrm{mL}$ streptomycin at $37^{\circ} \mathrm{C}$ in a $5 \% \mathrm{CO}_{2}$ atmosphere. HPCs were transiently transfected at $10^{6}$ cells per well using FuGENE HD Transfection Reagent (Promega) according to the manufacturer's instructions. Briefly, the reagent was premixed with the various plasmids at a ratio of $1.0 \mu \mathrm{g}$ DNA to $3 \mu \mathrm{L}$ transfection reagents in serum-free DMEM and incubated for $15 \mathrm{~min}$ at room temperature, and then, the mixture was added to the cells cultured in six-well plates. After $6 \mathrm{~h}$, the medium was replaced with normal culture medium and incubated for another $24 \mathrm{~h}$. Western blotting was used to detect the transfection efficiency.

\section{Western blotting and Co-IP}

Proteins were extracted from HPCs using RIPA buffer (Solarbio, Beijing, China), separated by $10 \%$ SDS-PAGE, and transferred to a PVDF membrane (Millipore, Burlington, MA). The membrane was blocked with $5 \%$ nonfat milk and incubated with the primary antibodies overnight at $4^{\circ} \mathrm{C}$. After washing with TBS-T buffer $(50 \mathrm{~m} M$ Tris- $\mathrm{HCl}$ [pH 7.4], $100 \mathrm{~m} M \mathrm{NaCl}, 0.5 \%$ Tween 20), the membranes were incubated with goat anti-rabbit or mouse secondary antibody conjugated to horseradish peroxidase. After washing, bands 
were detected by ECL reagent and scanned using the Odyssey Fc System (LI-COR, Lincoln, NE).

For Co-IP, cells were lysed with lysis buffer ( $50 \mathrm{~m} M$ Tris/ $\mathrm{HCl}, 150 \mathrm{~m} M \mathrm{NaCl}, 1 \mathrm{~m} M$ EDTA, $0.25 \%$ sodium deoxycholate, $1 \%$ NP-40, $1 \mathrm{mM}$ PMSF, $2 \mathrm{mg} / \mathrm{mL}$ aprotinin, $\mathrm{pH}$ 7.4) on ice for $30 \mathrm{~min}$ and centrifuged at $12,000 \mathrm{rpm}$ for $20 \mathrm{~min}$. The supernatant was incubated with the appropriate primary antibodies overnight and followed by the addition of protein A/G-Sepharose (Santa Cruz, Dallas, TX) at $4^{\circ} \mathrm{C}$ for $3 \mathrm{~h}$. After three washes with homogenization buffer, the beads were boiled with a $6 \times$ buffer for 5 min followed by SDS-PAGE and Western blotting using the appropriate antibodies. The protein bands were detected by ECL detection system.

\section{$R T-q P C R$ analysis}

We extracted total RNA from HPCs by using TRIzol reagent and RT-qPCR kits according to the manufacturer's guidelines. Complementary DNA was amplified with specific primers by means of PCR for nephrin, synaptopodin, and 18s rRNA. SYBR Premix Ex Taq II was used to perform realtime PCR, and real-time PCRs were processed on an Agilent Mx3000P qPCR Systems (Agilent, CA). Primers were as follows: human synaptopodin (forward 5'-CTTACGGC GGTGACATCTC-3', reverse 5'-GGTCCTGAGCCTCGA TCC-3') and human nephrin (forward 5'-AGCTCGTG TCTCCCAGAGT-3', reverse 5'-CGTTCACGTTTGCAG AGATGT-3').

\section{In vitro Cdk5 kinase activity assay}

The protein G Plus/protein A-agarose beads with Cdk5 protein was incubated with $6 \mu \mathrm{L}$ of assay dilution buffer containing $10 \mu \mathrm{g}$ of histone H1 (14-155; Millipore Sigma, Burlington, MA) and $12 \mu \mathrm{L}$ of magnesium/ATP mixture (20113; Millipore Sigma) in a final volume of $30 \mu \mathrm{L}$ at $30^{\circ} \mathrm{C}$ for $30 \mathrm{~min}$. The binding strength of phospho-histone $\mathrm{H} 1$ was assessed by immunoblotting with specific anti-phosphohistone H1 antibody (06-597; Millipore Sigma) (31).

\section{Mitochondrial morphology}

The mitochondrial morphology was observed by MitoTracker Deep Red FM staining. After treatment, cells were incubated with prewarmed $\left(37^{\circ} \mathrm{C}\right)$ staining solution $(50 \mathrm{n} M)$ for $30 \mathrm{~min}$ at $37^{\circ} \mathrm{C}$ in the dark. The stained cells washed with prewarmed phosphate-buffered saline (PBS) buffer and observed with a laser confocal microscope (Leica, Germany).

\section{Mitochondrial ROS detection and flow cytometry}

The mitochondrial formation of ROS was detected using MitoSOX Red. After culture in six-well plates under the different experimental conditions for $48 \mathrm{~h}$, cells were incubated with MitoSOX Red at a final concentration of $5 \mu M$ for $30 \mathrm{~min}$ at $37^{\circ} \mathrm{C}$. Cells were washed twice with PBS, and images were acquired using a laser confocal microscope (Leica). The mitochondrial ROS intensity was quantified using the Image-Pro Plus 6.0 software.

For flow cytometry, cells were incubated with DCFH-DA probe $(10 \mu \mathrm{M})$ for $30 \mathrm{~min}$ at $37^{\circ} \mathrm{C}$ in the dark incubator, analyzed with an FACS flow cytometer (FACS Aria, BD Biosciences, $\mathrm{CA}$ ) at specific excitation and emission wave- lengths (488 and $525 \mathrm{~nm}$, respectively). The mean fluorescence intensity (MFI) of $\sim 1 \times 10^{4}$ cells was analyzed using the FlowJo software (27).

\section{$\Delta \Psi m$ assay}

Cells were seeded on a six-well plate, treated for indicated time, and then washed twice with PBS before incubation with $5 \mu \mathrm{M} \mathrm{JC}-1$ probe at $37^{\circ} \mathrm{C}$ in the dark for $30 \mathrm{~min}$. After thorough washing, the cells were subjected to FACS flow cytometer or detected by a fluorospectrophotometer. Results of flow cytometry were analyzed using the FlowJo software (53). Images were acquired using a laser confocal microscope (Leica, Germany). The $\Delta \Psi \mathrm{m}$ intensity was quantified using the Image-Pro Plus 6.0 software.

\section{Intracellular ATP content determination}

ATP levels were assessed using an ATP Determination Kit (Beyotime Company, China). For this, cells were harvested and lysed with lysis reagent. After centrifugation at 12,000 $\mathrm{g}$ for $20 \mathrm{~min}$ at $4^{\circ} \mathrm{C}$, the supernatant was collected, and the ATP levels were detected according to the manufacturer's instructions. Cellular ATP levels were expressed as a percentage of the levels observed in control cells.

\section{Senescence $\beta$-galactosidase staining}

Detection of SA- $\beta$-Gal-positive cells was conducted using a kit (Beyotime, Shanghai, China) according to the manufacturer's instructions. Briefly, HPCs were seeded in six-well plates at a cell density of $5.0 \times 10^{4}$ cells per well containing complete medium and incubated at $37^{\circ} \mathrm{C}$ for $24 \mathrm{~h}$. Cells were washed in PBS and fixed with $2 \%$ formaldehyde and then incubated with SA- $\beta$-gal staining solution at $37^{\circ} \mathrm{C}$. After $24 \mathrm{~h}$ incubation, the "blue" cells expressing SA- $\beta$-Gal were counted under the microscope.

\section{Immunohistochemistry}

Immunohistochemistry for antibodies on renal sections was performed with SP kit according to the instructions of the manufacturer. Paraffin-embedded tissue sections $(4 \mu \mathrm{m})$ were deparaffinized in xylene and rehydrated with graded ethanol. Internal peroxidase was inactivated with $3 \%$ hydrogen peroxide in $100 \%$ methanol for $30 \mathrm{~min}$. Antigen retrieval was subsequently performed by autoclaving for $5 \mathrm{~min}$ at $121^{\circ} \mathrm{C}$ in a sodium citrate buffer ( $\mathrm{pH}$ 6.0). After blocking with $10 \%$ normal goat serum for $30 \mathrm{~min}$ at $37^{\circ} \mathrm{C}$, the sections were incubated with primary antibodies for Cdk5, nephrin, synaptopodin, Sirt1, p-Sirt1, Drp1, p-Drp1, Mfn1, or CytoC overnight at $4^{\circ} \mathrm{C}$. The next day, sections were washed with PBS and incubated with secondary antibody for $30 \mathrm{~min}$ at $37^{\circ} \mathrm{C}$. Labeling was visualized with 3,3-diaminobenzidine to produce a brown color, and sections were counterstained with hematoxylin.

\section{Immunofluorescence}

Human podocytes were grown and stimulated in six-well chamber slides and fixed with frozen acetone for $10 \mathrm{~min}$ at $4^{\circ} \mathrm{C}$. The cell membranes were punched with Triton X-100 before incubation with anti-Cdk5, anti-nephrin, antisynaptopodin, anti-Sirt1, or anti-p-Sirt1 antibody at $4^{\circ} \mathrm{C}$ 
overnight. The cells were then exposed to goat anti-rabbit Alexa Fluor 552-conjugated secondary antibody for $1 \mathrm{~h}$ at $37^{\circ} \mathrm{C}$. Immunofluorescence of cells was then examined by laser confocal microscopy.

\section{Transmission electron microscopy}

Renal tissues of diabetic mice were perfused with PBS and cut into $1 \mathrm{~mm}^{3}$ blocks, which were immediately fixed with 4\% glutaraldehyde. After embedding in epoxy resin, the ultrathin tissue sections were stained with uranyl acetate and lead citrate and then examined with Hitachi H7650 transmission electron microscope and photographed. Images were analyzed with the ImageJ software. Quantification of mitochondria was performed as described previously. The morphology of a total 100 mitochondria was examined for all the groups.

\section{Statistical analysis}

All experiments were performed at least three separate times, with data obtained from triplicate wells in each experiment. Data are expressed as means \pm SD. The statistical difference between the two groups was analyzed by an unpaired Student's $t$-test (two-tailed); multiple groups were compared using one-way analysis of variance and correlated by the Spearman correlation analysis (GraphPad Prism 5.0, Bethesda, MD). A value of $p<0.05$ was considered significant.

\section{Author Disclosure Statement}

No competing financial interests exist.

\section{Funding Information}

This work was supported by the National Natural Science Foundation of China (81770720), the Natural Science Foundation of Hebei Province (H2019206549 and H2019206550), the Key Project of Scientific and Technological Research of Hebei Province (ZD2018003), the People's Livelihood Science and Technology Project of Hebei Province (19277784D), and the Graduate Innovation Funding Project of Hebei Province (CXZZBS2019107).

\section{Supplementary Material}

Supplementary Figure S1

Supplementary Figure S2

Supplementary Figure S3

Supplementary Figure S4

Supplementary Figure S5

Supplementary Figure S6

Supplementary Figure S7

Supplementary Figure S8

Supplementary Figure S9

Supplementary Figure S10

Supplementary Figure S11

\section{References}

1. Ahmed HH, Taha FM, Omar HS, Elwi HM, and Abdelnasser M. Hydrogen sulfide modulates SIRT1 and suppresses oxidative stress in diabetic nephropathy. Mol Cell Biochem 457: 1-9, 2019.
2. Alcendor RR, Gao S, Zhai P, Zablocki D, Holle E, Yu X, Tian B, Wagner T, Vatner SF, and Sadoshima J. Sirt1 regulates aging and resistance to oxidative stress in the heart. Circ Res 100: 1512-1521, 2007.

3. Aquilano K, Vigilanza P, Baldelli S, Pagliei B, Rotilio G, and Ciriolo MR. Peroxisome proliferator-activated receptor gamma co-activator 1alpha (PGC-1alpha) and sirtuin 1 (SIRT1) reside in mitochondria: possible direct function in mitochondrial biogenesis. J Biol Chem 285: 21590-21599, 2010.

4. Bai B, Liang Y, Xu C, Lee MY, Xu A, Wu D, Vanhoutte PM, and Wang Y. Cyclin-dependent kinase 5-mediated hyperphosphorylation of sirtuin-1 contributes to the development of endothelial senescence and atherosclerosis. Circulation 126: 729-740, 2012.

5. Bible E. Diabetic nephropathy: Sirt1 attenuates diabetic albuminuria. Nat Rev Nephrol 9: 696, 2013.

6. Brinkkoetter PT, Bork T, Salou S, Liang W, Mizi A, Ozel C, Koehler S, Hagmann HH, Ising C, Kuczkowski A, Schnyder S, Abed A, Schermer B, Benzing T, Kretz O, Puelles VG, Lagies S, Schlimpert M, Kammerer B, Handschin C, Schell C, and Huber TB. Anaerobic glycolysis maintains the glomerular filtration barrier independent of mitochondrial metabolism and dynamics. Cell Rep 27: 1551-1566 e5, 2019.

7. Brinkkoetter PT, Wu JS, Ohse T, Krofft RD, Schermer B, Benzing T, Pippin JW, and Shankland SJ. p35, the noncyclin activator of $\mathrm{Cdk} 5$, protects podocytes against apoptosis in vitro and in vivo. Kidney Int 77: 690-699, 2010.

8. Camins A, Verdaguer E, Folch J, Canudas AM, and Pallas M. The role of CDK5/P25 formation/inhibition in neurodegeneration. Drug News Perspect 19: 453-460, 2006.

9. Chau MD, Gao J, Yang Q, Wu Z, and Gromada J. Fibroblast growth factor 21 regulates energy metabolism by activating the AMPK-SIRT1-PGC-1alpha pathway. Proc Natl Acad Sci U S A 107: 12553-12558, 2010.

10. Cheng K and Ip NY. Cdk5: a new player at synapses. Neurosignals 12: 180-190, 2003.

11. Choi SE, Kwon S, Seok S, Xiao Z, Lee KW, Kang Y, Li X, Shinoda K, Kajimura S, Kemper B, and Kemper JK. Obesity-linked phosphorylation of SIRT1 by Casein Kinase 2 inhibits its nuclear localization and promotes fatty liver. Mol Cell Biol 37: e00006-17, 2017.

12. Conrad E, Polonio-Vallon T, Meister M, Matt S, Bitomsky N, Herbel C, Liebl M, Greiner V, Kriznik B, Schumacher $S$, Krieghoff-Henning E, and Hofmann TG. HIPK2 restricts SIRT1 activity upon severe DNA damage by a phosphorylation-controlled mechanism. Cell Death Differ 23: 110-122, 2016.

13. Duann $\mathrm{P}$ and Lin PH. Mitochondria Damage and Kidney Disease. Adv Exp Med Biol 982: 529-551, 2017.

14. Du YG, Zhang KN, Gao ZL, Dai F, Wu XX, and Chai KF. Tangshen formula improves inflammation in renal tissue of diabetic nephropathy through SIRT1/NF- $\kappa$ B pathway. Exp Ther Med 15: 2156-2164, 2018.

15. Eid AA, Gorin Y, Fagg BM, Maalouf R, Barnes JL, Block $\mathrm{K}$, and Abboud HE. Mechanisms of podocyte injury in diabetes: role of cytochrome P450 and NADPH oxidases. Diabetes 58: 1201-1211, 2009.

16. Farmer T, Naslavsky N, and Caplan S. Tying trafficking to fusion and fission at the mighty mitochondria. Traffic 19: 569-577, 2018.

17. Galvan DL, Long J, Green N, Chang BH, Lin JS, Schumacker $\mathrm{P}$, Truong LD, Overbeek $\mathrm{P}$, and Danesh FR. 
Drp1S600 phosphorylation regulates mitochondrial fission and progression of nephropathy in diabetic mice. J Clin Invest 129: 2807-2823, 2019.

18. Guo MY, Shang L, Hu YY, Jiang LP, Wan YY, Zhou QQ, Zhang K, Liao HF, Yi JL, and Han XJ. The role of Cdk5mediated Drp1 phosphorylation in Abeta1-Abeta42 induced mitochondrial fission and neuronal apoptosis. $J$ Cell Biochem 119: 4815-4825, 2018.

19. Gupta KK and Singh SK. Cdk5: a main culprit in neurodegeneration. Int J Neurosci 129: 1192-1197, 2019.

20. Hong Q, Zhang L, Das B, Li Z, Liu B, Cai G, Chen X, Chuang PY, He JC, and Lee K. Increased podocyte Sirtuin1 function attenuates diabetic kidney injury. Kidney Int 93: 1330-1343, 2018.

21. Horne SJ, Vasquez JM, Guo Y, Ly V, Piret SE, Leonardo AR, Ling J, Revelo MP, Bogenhagen D, Yang VW, He JC, and Mallipattu SK. Podocyte-specific loss of Kruppel-like factor 6 increases mitochondrial injury in diabetic kidney disease. Diabetes 67: 2420-2433, 2018.

22. Jang WB, Park JH, Ji ST, Lee NK, Kim DY, Kim YJ, Jung SY, Kang S, Lamichane S, Lamichane BD, Ha J, Yun J, Moon HR, Baek SH, Chung HY, and Kwon SM. Cytoprotective roles of a novel compound, MHY-1684, against hyperglycemia-induced oxidative stress and mitochondrial dysfunction in human cardiac progenitor cells. Oxid Med Cell Longev 2018: 4528184, 2018.

23. Kumar GS, Kulkarni A, Khurana A, Kaur J, and Tikoo K. Selenium nanoparticles involve HSP-70 and SIRT1 in preventing the progression of type 1 diabetic nephropathy. Chem Biol Interact 223: 125-133, 2014.

24. Kumazawa A, Mita N, Hirasawa M, Adachi T, Suzuki H, Shafeghat N, Kulkarni AB, Mikoshiba K, Inoue T, and Ohshima T. Cyclin-dependent kinase 5 is required for normal cerebellar development. Mol Cell Neurosci 52: 97-105, 2013.

25. Lee J, Hyon JY, Min JY, Huh YH, Kim HJ, Lee H, Yun $\mathrm{SH}$, Choi CW, Jeong Ha S, Park J, Chung YH, Jeong HG, Ha SK, Jung SK, Kim Y, and Han EH. Mitochondrial carnitine palmitoyltransferase 2 is involved in N(epsilon)(carboxymethyl)-lysine-mediated diabetic nephropathy. Pharmacol Res 152: 104600, 2019.

26. Lee SE, Jang JE, Kim HS, Jung MK, Ko MS, Kim MO, Park HS, Oh W, Choi SJ, Jin HJ, Kim SY, Kim YJ, Kim SW, Kim MK, Sung CO, Pack CG, Lee KU, and Koh EH. Mesenchymal stem cells prevent the progression of diabetic nephropathy by improving mitochondrial function in tubular epithelial cells. Exp Mol Med 51: 77, 2019.

27. Li Q, Dong Z, Lian W, Cui J, Wang J, Shen H, Liu W, Yang J, Zhang X, and Cui H. Ochratoxin A causes mitochondrial dysfunction, apoptotic and autophagic cell death and also induces mitochondrial biogenesis in human gastric epithelium cells. Arch Toxicol 93: 1141-1155, 2019.

28. Lin YC, Chang YH, Yang SY, Wu KD, and Chu TS. Update of pathophysiology and management of diabetic kidney disease. J Formos Med Assoc 117: 662-675, 2018.

29. Liu R, Zhong Y, Li X, Chen H, Jim B, Zhou MM, Chuang $\mathrm{PY}$, and He JC. Role of transcription factor acetylation in diabetic kidney disease. Diabetes 63: 2440-2453, 2014.

30. Liu W, Zhang Y, Hao J, Liu S, Liu Q, Zhao S, Shi Y, and Duan H. Nestin protects mouse podocytes against high glucose-induced apoptosis by a Cdk5-dependent mechanism. J Cell Biochem 113: 3186-3196, 2012.

31. Liu W, Zhou Y, Liang R, and Zhang Y. Inhibition of cyclin-dependent kinase 5 activity alleviates diabetesrelated cognitive deficits. FASEB J 33: 14506-14515, 2019.
32. Ma Y, Chen Z, Tao Y, Zhu J, Yang H, Liang W, and Ding $\mathrm{G}$. Increased mitochondrial fission of glomerular podocytes in diabetic nephropathy. Endocr Connect 8: 1206-1212, 2019.

33. Marshall CB and Shankland SJ. Cell cycle regulatory proteins in podocyte health and disease. Nephron Exp $\mathrm{Ne}$ phrol 106: e51-e59, 2007.

34. Morigi M, Perico L, and Benigni A. Sirtuins in renal health and disease. J Am Soc Nephrol 29: 1799-1809, 2018.

35. Morishima M, Horikawa K, and Funaki M. Cardiomyocytes cultured on mechanically compliant substrates, but not on conventional culture devices, exhibit prominent mitochondrial dysfunction due to reactive oxygen species and insulin resistance under high glucose. PLoS One 13: e0201891, 2018.

36. Nikhil K and Shah K. The Cdk5-Mcl-1 axis promotes mitochondrial dysfunction and neurodegeneration in a model of Alzheimer's disease. J Cell Sci 130: 3023-3039, 2017.

37. Pagtalunan ME, Miller PL, Jumping-Eagle S, Nelson RG, Myers BD, Rennke HG, Coplon NS, Sun L, and Meyer TW. Podocyte loss and progressive glomerular injury in type II diabetes. J Clin Invest 99: 342-348, 1997.

38. Papadopoulou-Marketou N, Paschou SA, Marketos N, Adamidi S, Adamidis S, and Kanaka-Gantenbein C. Diabetic nephropathy in type 1 diabetes. Minerva Med 109: 218-228, 2018.

39. Pavenstadt H, Kriz W, and Kretzler M. Cell biology of the glomerular podocyte. Physiol Rev 83: 253-307, 2003.

40. Song H, Kim W, Choi JH, Kim SH, Lee D, Park CH, Kim S, Kim DY, and Kim KT. Stress-induced nuclear translocation of CDK5 suppresses neuronal death by downregulating ERK activation via VRK3 phosphorylation. Sci Rep 6: 28634, 2016.

41. Su M, Dhoopun AR, Yuan Y, Huang S, Zhu C, Ding G, Liu B, Yang T, and Zhang A. Mitochondrial dysfunction is an early event in aldosterone-induced podocyte injury. Am J Physiol Renal Physiol 305: F520-F531, 2013.

42. Sun Z, Ma Y, Chen F, Wang S, Chen B, and Shi J. miR$133 \mathrm{~b}$ and miR-199b knockdown attenuate TGF- $\beta 1$-induced epithelial to mesenchymal transition and renal fibrosis by targeting SIRT1 in diabetic nephropathy. Eur J Pharmacol 837: 96-104, 2018.

43. Susztak K, Raff AC, Schiffer M, and Bottinger EP. Glucose-induced reactive oxygen species cause apoptosis of podocytes and podocyte depletion at the onset of diabetic nephropathy. Diabetes 55: 225-233, 2006.

44. Taniguchi Y, Pippin JW, Hagmann H, Krofft RD, Chang AM, Zhang J, Terada Y, Brinkkoetter P, and Shankland SJ. Both cyclin I and p35 are required for maximal survival benefit of cyclin-dependent kinase 5 in kidney podocytes. Am J Physiol Renal Physiol 302: F1161-F1171, 2012.

45. Teiken JM, Audettey JL, Laturnus DI, Zheng S, Epstein PN, and Carlson EC. Podocyte loss in aging OVE26 diabetic mice. Anat Rec (Hoboken) 291: 114-121, 2008.

46. Wang J, Dong ZH, Gui MT, Yao L, Li JH, Zhou XJ, and Fu DY. HuoXue QianYang QuTan Recipe attenuates left ventricular hypertrophy in obese hypertensive rats by improving mitochondrial function through SIRT1/PGC-1alpha deacetylation pathway. Biosci Rep 39: BSR20192909, 2019.

47. Wang W, Wang Y, Long J, Wang J, Haudek SB, Overbeek P, Chang BH, Schumacker PT, and Danesh FR. Mitochondrial fission triggered by hyperglycemia is mediated by ROCK1 activation in podocytes and endothelial cells. Cell Metab 15: 186-200, 2012. 
48. Wang XL, Wu LY, Zhao L, Sun LN, Liu HY, Liu G, and Guan GJ. SIRT1 activator ameliorates the renal tubular injury induced by hyperglycemia in vivo and in vitro via inhibiting apoptosis. Biomed Pharmacother 83: 41-50, 2016.

49. Wei PZ and Szeto CC. Mitochondrial dysfunction in diabetic kidney disease. Clin Chim Acta 496: 108-116, 2019.

50. Wikstrom JD, Mahdaviani K, Liesa M, Sereda SB, Si Y, Las G, Twig G, Petrovic N, Zingaretti C, Graham A, Cinti S, Corkey BE, Cannon B, Nedergaard J, and Shirihai OS. Hormone-induced mitochondrial fission is utilized by brown adipocytes as an amplification pathway for energy expenditure. EMBO J 33: 418-436, 2014.

51. Yang S, Han Y, Liu J, Song P, Xu X, Zhao L, Hu C, Xiao L, Liu F, Zhang H, and Sun L. Mitochondria: a novel therapeutic target in diabetic nephropathy. Curr Med Chem 24: 3185-3202, 2017.

52. Ye T, Fu AK, and Ip NY. Cyclin-dependent kinase 5 in axon growth and regeneration. Int Rev Neurobiol 105: 91$115,2012$.

53. Yoshizaki T, Milne JC, Imamura T, Schenk S, Sonoda N, Babendure JL, Lu JC, Smith JJ, Jirousek MR, and Olefsky JM. SIRT1 exerts anti-inflammatory effects and improves insulin sensitivity in adipocytes. Mol Cell Biol 29: 13631374, 2009.

54. Zeb A, Kim D, Alam SI, Son M, Kumar R, Rampogu S, Parameswaran S, Shelake RM, Rana RM, Parate S, Kim JY, and Lee KW. Computational simulations identify pyrrolidine-2,3-dione derivatives as novel inhibitors of Cdk5/p25 complex to attenuate Alzheimer's Pathology. $J$ Clin Med 8: 746, 2019.

55. Zhang Y, Li H, Hao J, Zhou Y, and Liu W. High glucose increases Cdk5 activity in podocytes via transforming growth factor-beta1 signaling pathway. Exp Cell Res 326: 219-229, 2014.

56. Zhang Z, Zhao R, Tang Y, Wen S, Wang D, and Qi J. Fuzhisan, a Chinese herbal medicine, inhibits betaamyloid-induced neurotoxicity and tau phosphorylation through calpain/Cdk5 pathway in cultured cortical neurons. Neurochem Res 36: 801-811, 2011.

57. Zheng YL, Zhang X, Fu HX, Guo M, Shukla V, Amin ND, E J, Bao L, Luo HY, Li B, Lu XH, and Gao YC. Knockdown of Expression of Cdk5 or p35 (a Cdk5 Activator) Results in Podocyte Apoptosis. PLoS One 11: e0160252, 2016.

58. Zhou D, Zhou M, Wang Z, Fu Y, Jia M, Wang X, Liu M, Zhang Y, Sun Y, Lu Y, Tang W, and Yi F. PGRN acts as a novel regulator of mitochondrial homeostasis by facilitating mitophagy and mitochondrial biogenesis to prevent podocyte injury in diabetic nephropathy. Cell Death Dis 10: $524,2019$.
Address correspondence to:

Dr. Huijun Duan

Key Laboratory of Kidney Diseases of Hebei Province

Department of Pathology

Hebei Medical University

No. 361 Zhongshan East Road

Shijiazhuang 050017

China

E-mail: huijunduan2020@163.com

Prof. Wei Liu

Key Laboratory of Kidney Diseases of Hebei Province

Department of Pathology

Hebei Medical University

No. 361 Zhongshan East Road

Shijiazhuang 050017

China

E-mail: weiliu0209@hebmu.edu.cn

Date of first submission to ARS Central, January 19, 2020; date of final revised submission, July 3, 2020; date of acceptance, July 6, 2020.

$\begin{aligned} & \text { Abbreviations Used } \\ \Delta \Psi \mathrm{M} & =\text { mitochondrial transmembrane potential } \\ \mathrm{AD} & =\text { Alzheimer's disease } \\ \mathrm{ATP} & =\text { adenosine triphosphate } \\ \mathrm{BUN} & =\text { blood urea nitrogen } \\ \mathrm{Cdk} 5 & =\text { cyclin-dependent kinase } 5 \\ \mathrm{Co}-\mathrm{IP} & =\text { co-immunoprecipitation } \\ \mathrm{DN} & =\text { diabetic nephropathy } \\ \mathrm{FPs} & =\text { foot processes } \\ \mathrm{GBM} & =\text { glomerular basement membrane } \\ \mathrm{HG} & =\text { high glucose } \\ \mathrm{HPC} & =\text { human renal podocyte } \\ \mathrm{NAC} & =\text { N-acetyl-L-cysteine } \\ \mathrm{PBS} & =\text { phosphate-buffered saline } \\ \mathrm{PD} & =\text { Parkinson's disease } \\ \mathrm{ROS} & =\text { reactive oxygen species } \\ \mathrm{RSV} & =\text { resveratrol } \\ \mathrm{RT}-\mathrm{qPCR}= & \text { quantitative real-time polymerase chain } \\ \mathrm{Sirt} 1 & =\text { Sirtuin } 1 \\ \mathrm{STZ} & =\text { streptozotocin } \\ \mathrm{TGF}-\beta 1 & =\text { transforming growth factor- } \beta 1\end{aligned}$

